Flow in the thin film created by a turbulent water jet impinging on a vertical wall R.K. Bhagat and D.I. Wilson

Department of Chemical Engineering and Biotechnology, New Museums Site, Pembroke Street, Cambridge, CB2 3RA, UK

\author{
Submitted to \\ Chemical Engineering Science \\ CES-D-16-00399 \\ Revised Manuscript \\ (C) RKB \& DiW
}
Corresponding Author
D. Ian Wilson
Department of Chemical Engineering and Biotechnology
New Museums Site
Pembroke Street
Cambridge
CB2 3RA
UK

$\begin{array}{ll}\text { Tel } & +441223334791 \\ \text { Fax } & +441223334796 \\ \text { E-mail } & \text { diw11@cam.ac.uk }\end{array}$




\title{
Flow in the thin film created by a coherent turbulent water jet impinging on a vertical wall
}

\author{
R.K. Bhagat and D.I. Wilson \\ Department of Chemical Engineering and Biotechnology, New Museums Site, Pembroke \\ Street, Cambridge, CB2 3RA, UK
}

\section{Abstract}

When a liquid jet impinges on a vertical wall it forms a thin film which flows radially away from the point of impingement until a point where the outward momentum is balanced by surface tension and a film jump is formed. The model for the location for the film jump presented by Wilson et al. (Chem. Eng. Sci, 2012, Vol. 68, pp 449-460) is revised to include the development of laminar and turbulent boundary layers in the thin film. The criterion for film jump formation is also revisited, and the analysis explains why the location is insensitive to the nature of the wall material at high flow rates. The model is compared with published data for velocity profiles in the thin film, the transition to turbulence, and new experimental data where the average velocity in the thin film was estimated from the initial growth of the radial flow pattern for flow rates of 1.95 to $4.01 \mathrm{dm}^{3} \mathrm{~s}^{-1}$, corresponding to jet Reynolds numbers of 15500 to 32000 . Very good agreement with the published and measured data is obtained, with no adjustable parameters, for jets impinging perpendicularly as well as at an oblique angle. The model shows that the parabolic velocity profile assumed by Wilson et al. gives a reasonable estimate of the average velocity, but it is not able to predict phenomena such as the observed transition to turbulence.

Keywords: Boundary layer; contact angle; film jump; impinging jet; oblique jet; thin film

\section{Introduction}

Liquid jets are widely used for cleaning, with applications ranging from the internal and external surfaces of processing and storage vessels (Burfoot and Middleton, 2009), to kitchenware in dishwashers (Pérez-Mohedano et al., 2015). Liquid jets impinging on solid surfaces are also employed in process intensification for enhancing local heat transfer rates (Lienhard, 1995). High speed jets can also be used for cutting (Leach and Walker, 1966). In 
cleaning applications, knowledge of the flow pattern created by the jet is important for determining local cleaning rates as well as predicting wetting and draining behaviour.

Following impingement, the liquid in the jet spreads radially away from the point of impingement in a thin film until the height of the film changes abruptly. When a coherent vertical jet impinges perpendicularly downwards on a horizontal surface the transition, termed the hydraulic jump, is circular (Watson, 1964). A vertical jet impinging upwards gives rise to a circular wetted region bounded by falling droplets or a liquid curtain known as the water bell (Button et al., 2010).

Many cleaning applications involve walls which are close to vertical and the influence of gravity gives rise to less symmetric flow patterns. When a coherent horizontal jet impinges on a vertical wall, as shown in Figure 1, the liquid initially spreads out in a thin film in a region which is here labelled the radial flow zone (RFZ). At some radial position the outwards momentum in the film is countered by surface tension and a jump is observed: it is here termed the film jump to differentiate it from the hydraulic jump. Gravity causes the location of the film jump to vary with azimuthal position, $\theta$. Beyond the film jump, the liquid drains under gravity and flows circumferentially around the perimeter of the RFZ in the rope. Below the level of impingement (marked $\mathrm{AA}^{\prime}$ on Figure 1), the rope and radial flow spread out further to generate a falling film which may narrow further downstream.

The hydrodynamics of the symmetric, circular hydraulic jump have been studied in detail. The transition from a fast moving film with a developing boundary layer to a laminar film and subsequently a turbulent film has been analysed mathematically and investigated experimentally (e.g. Watson, 1964; Bohr et al., 1993; Bush and Aristoff, 2003; Arakeri and Rao, 2013). Similar analyses have also been applied to inclined jets (Blyth and Pozrikidis, 2005; Kate et al., 2007).

Morison and Thorpe (2002) reported the first systematic study of jets impinging on vertical walls. Wilson et al. (2012) presented a model for the flow in the RFZ which gave good agreement with Morison and Thorpe's data for the width at $\mathrm{AA}^{\prime}$, marked $R_{\mathrm{j}, 90}$ in Figure 1 , as well as new experimental data sets. The dimensions of the RFZ for obliquely impinging jets and the behaviour below have since been investigated by Wilson and co-workers (Wilson et 
al., 2012; Wang et al., 2013a,b; Wang et al., 2015; Aouad et al., 2016) and by other groups (e.g. Gordeev et al., 2015).

In the model of Wilson et al. (2012) the flow in the film is treated as laminar, with a parabolic velocity profile similar to that in Nusselt's analysis of film condensation (Nusselt, 1916). The location of the film jump was obtained from a balance between the outward flow of momentum in the liquid and the retarding force provided by surface tension. The agreement between their model and experimental data at lower flow rates $\left(<10 \mathrm{~g} \mathrm{~s}^{-1}\right)$ was very good; at higher flow rates, approaching those of interest for industrial cleaning applications, their model tended to overpredict the location of the film jump (Wang et al., 2013a). Their model included a contribution from the wall material, via the contact angle $\beta$, which was not observed experimentally at higher flow rates: moreover, similar RFZ dimensions were found on walls with different wetting characteristics.

At higher flow rates the liquid film is likely to enter the turbulent regime (see Azuma and Hoshino, 1984a,b; Lienhard, 2006) and the Nusselt film behaviour assumed by Wilson et al. (2012) is unlikely to be valid for the whole film. The aim of this study was to revisit the treatment of the flow of the liquid film in the RFZ for horizontal (and inclined) jets impinging on vertical walls, incorporating the effect of turbulence in the film. The analysis is guided by the approaches taken to describe hydraulic jumps. The results are compared with data reported in previous studies in the literature as well as new estimates of the average velocity in the film obtained from observation of the early stages of jet impingement. Several features of the flow in the RFZ, including the transitions in wave behaviour and heat transfer performance reported by Azuma and Hoshino (1984a,b); Liu et al., (1991) and Lienhard (1995) are compared with the results from the analysis.

The primary objective was to establish the limit of validity of the Wilson et al. (2012) model, in addition to providing a description for faster jets. Furthermore, a revisit of the criterion for the location of the film jump presented by Wilson et al. (2012) yields an explanation of why the film behaves as if the contact angle at the rope-wall boundary is effectively $\pi / 2$, independent of the nature of the wall material, at higher flow rates. 


\section{Model development}

Figure 1 shows a schematic of the flow profile created by normal impingement $\left(\phi=90^{\circ}\right)$ of a coherent liquid jet on a vertical surface. The key regions are labelled: $(i)$ the radial flow zone (RFZ); (ii) the boundary of the radial flow zone, the film jump; (iii) the rope region; and (iv) the draining film. The RFZ is important for cleaning applications as the wall shear stress and momentum in the film are greatest in this region.

The model is presented in three sections. The first section deals with the flow in the film for a jet impinging perpendicularly on a vertical wall. The second considers the formation of the film jump. The third considers the case where the jet impinges obliquely $\left(\phi<90^{\circ}\right.$ or $\left.\phi>90^{\circ}\right)$.

\subsection{Normal impingement of a jet on a vertical surface}

For the range of flow rates used in the present analysis, the velocity component in the azimuthal direction, $v_{\theta}$, arising due to gravitational acceleration is small compared to the radial component of velocity $U_{a v}$ (see Appendix I). Therefore ignoring the component of velocity in the azimuthal direction and applying the boundary layer approximation for a twodimensional film flow, the governing differential equations for an axisymmetric film flowing radially away from the point of impingement are:

$$
\begin{array}{ll}
\frac{1}{r} \frac{\partial(u r)}{\partial r}+\frac{\partial v}{\partial z}=0 & \text { (Continuity) } \\
u \frac{\partial u}{\partial r}+v \frac{\partial u}{\partial z}=-\frac{1}{\rho} \frac{\partial P}{\partial r}+\frac{\mu}{\rho} \frac{\partial^{2} u}{\partial z^{2}}-g \cos \theta & \text { (Navier-Stokes) }
\end{array}
$$

Cylindrical co-ordinates are used: $r$ is the radial co-ordinate; $z$ is the co-ordinate normal to the plane; $\theta$ is the azimuthal angle; $P$ is the gauge pressure; $u$ and $v$ are the components of velocity in the $r$ and $z$ directions, respectively; $g$ is the gravitational acceleration; $\mu$ is the fluid viscosity and $\rho$ its density.

The film thickness at radial position $r$ is $h$. The boundary conditions are

$$
\begin{array}{lll}
u=v=0 & \text { at } z=0 & \text { (No slip at wall) } \\
\frac{\partial u}{\partial z}=0 & \text { at } z=h & \text { (No shear stress at the free surface) }
\end{array}
$$

The volumetric flow rate in the jet is $Q=\pi d^{2} U_{\mathrm{o}} / 4$ where $U_{\mathrm{o}}$ is the mean velocity in the jet (assuming plug flow) and $d$ is the diameter of the jet (which is similar to the internal diameter of the nozzle, $d_{\mathrm{N}}$ ). Conservation of volume yields 


$$
Q=\int_{0}^{2 \pi} \int_{0}^{h} u d z r d \theta
$$

The jet Reynolds number, $R e_{\mathrm{j}} \equiv \rho d U_{\mathrm{o}} / \mu$, characterises the flow in the jet. The jets in the present study feature $6300<R e_{\mathrm{j}}<32000$, which lie in the turbulent regime: Landreth and Adrian (1990) discussed the criteria for liquid jets to be turbulent and used PIV measurements on impinging liquid jets to confirm the presence of turbulent characteristics in cases where $R e_{\mathrm{j}}=6560$.

The pressure gradient in radial direction, $\frac{\partial p}{\partial r}$, is set to zero (parallel streamlines). Integrating Equations (1) and (2) and applying Leibniz' rule yields the momentum integral equation

$$
\frac{1}{r} \frac{d}{d r} \int_{0}^{h} u^{2} r d z=-\left.\frac{\mu}{\rho} \frac{\partial u}{\partial z}\right|_{0}-h g \cos \theta
$$

In the following analysis, mirroring that of Azuma and Hoshino (1984b), the film flow is subdivided into three zones (see Figure 2): ( $i$ ) the boundary layer formation zone (BLFZ), (ii) the laminar zone (LZ), and (iii) the turbulent zone (TZ).

\section{Boundary layer formation zone}

In the BLFZ, it is assumed that the influence of the wall is restricted to within the growing boundary layer, of thickness $\delta$. Beyond the boundary layer the local velocity is initially that of the jet, $U_{\mathrm{o}}$ : this velocity is not influenced by the wall but is subject to gravitational acceleration, giving,

$$
U(r)=\sqrt{U_{0}^{2}-2 g r \cos \theta}
$$

Re-writing Equation (4) for the BLFZ gives

$$
\frac{1}{r} \frac{d}{d r} \int_{0}^{\delta} u^{2} r d z+\frac{1}{r} \frac{d}{d r} \int_{\delta}^{h} U^{2} r d z=-\left.\frac{\mu}{\rho} \frac{\partial u}{\partial z}\right|_{0}-h g \cos \theta
$$

Within the boundary layer, the velocity profile is assumed to be described by a similarity solution of the form

$$
u(r, z)=U(r) f(\eta)
$$

where $\eta=z / \delta$.

Substituting Equation (7) into (6) and integrating gives 
$\frac{1}{r} \frac{d}{d r}\left(U^{2} r \delta\right) \int_{0}^{1} f^{2}(\eta) d \eta+\frac{1}{r} \frac{d}{d r}\left(U^{2} r(h-\delta)\right)=-\left.\frac{\mu}{\rho} \frac{U}{\delta} f(\eta)^{\prime}\right|_{0}-$

$h g \cos \theta$

Writing

$$
\int_{0}^{1} f^{2}(\eta) d \eta=C_{1}
$$

and

$$
\left.f^{\prime}(\eta)\right|_{0}=C_{2}
$$

allows Equation (8) to be re-written as

$$
\left(C_{1}-1\right) \frac{1}{r} \frac{d}{d r}\left(U^{2} r \delta\right)+\frac{1}{r} \frac{d}{d r}\left(U^{2} r h\right)=-C_{2} \frac{\mu}{\rho} \frac{U}{\delta}-h g \cos \theta
$$

Applying conservation of volume (Equation (3)) to the two regions of the film gives

$$
Q=r \int_{0}^{2 \pi} \int_{0}^{\delta} u d z d \theta+r \int_{0}^{2 \pi} \int_{\delta}^{h} U d z d \theta
$$

For small values of $r$,

$$
\int_{0}^{2 \pi}\left(U_{o}^{2}-2 g r \cos \theta\right)^{0.5} d \theta \approx 2 \pi U_{o}
$$

Hence, Equation 10 can be written as,

$$
\frac{Q}{2 \pi}=r U_{o} \delta \int_{0}^{1} f(\eta) d \eta+r U_{o}(h-\delta)
$$

Writing $\int_{0}^{1} f(\eta) d \eta=C_{3}$ gives

$$
h=\frac{Q}{2 \pi r U_{o}}+\delta\left(1-C_{3}\right)
$$

Substituting Equation (13) into (9) yields

$$
\frac{d\left(\delta^{2}\right)}{d r}=-\frac{2 \delta^{2}}{r}-\frac{2 \delta^{2} g \cos \theta}{U^{2}} \frac{\left(1-2 C_{1}+C_{3}\right)}{\left(C_{1}-C_{3}\right)}-\frac{2 C_{2} \mu}{\rho U\left(C_{1}-C_{3}\right)}+\frac{Q g \cos \theta \delta}{\pi U_{o}\left(C_{1}-C_{3}\right) U^{2} r}
$$

For a given velocity profile, Equation (14) gives the thickness of the boundary layer at position $r$ and, from Equation (13), the film thickness, $h$.

In this analysis, the velocity profile in the boundary layer and in laminar region is assumed to be a fourth order polynomial in $z$, with

$$
u(r, z)=\begin{array}{cc}
U(r) & r>\delta \\
U(r) f(\eta) & 0<r \leq \delta
\end{array}
$$

where,

$$
f(\eta)=a_{1}+a_{2} \eta+a_{3} \eta^{2}+a_{4} \eta^{3}+a_{5} \eta^{4}
$$


Equation 16 must satisfy the following two boundary conditions;

$$
\begin{array}{ll}
f(\eta)=0 \text { at } \eta=0 & \text { No slip at solid boundary } \\
\left.f^{\prime}(\eta)\right|_{\eta=1}=0 & \text { Zero shear stress at open surface }
\end{array}
$$

Beyond the boundary layer, the fluid velocity is the surface velocity, giving

$$
f(\eta)=1 \quad \text { at } \eta=1 \quad \text { Surface velocity }
$$

The experimental measurements of the velocity profile in such thin films by Stevens and Webb (1993) show a strongly linear velocity profile bear the wall (see Supplementary Information S1) rather than a parabolic one (as used by Wilson et al. 2012) or a cubic (see Liu et al. 1991). The remaining two coefficients in Equation (16) were therefore found by setting the wall behaviour to be linear. This requires

$$
\begin{aligned}
& \int_{0}^{1} f(\eta)=\frac{1}{2} \\
& \left.f^{\prime}(\eta)\right|_{z=0}=1
\end{aligned}
$$

and yields the result.

$$
f(\eta)=\eta-\frac{3}{2} \eta^{2}+4 \eta^{3}-\frac{5}{2} \eta^{4}
$$

This velocity profile gave good agreement with the laser Doppler velocimetry results reported by Stevens and Webb (1993) (see Supplementary Information S1). It will also be shown later that the transition from smooth concentric waves to chaotic surface waves observed in experiments is described reasonably well by the model when the velocity profile in the developing film is described by Equation (22).

Equations (14), (15) and (22) are solved numerically for a given value of $\theta$ to give the local film thickness and velocity. For the case where $\theta=90^{\circ}$, gravity has no effect and an analytical solution to (14) can be obtained, viz.

$$
\delta=2.12 \sqrt{\frac{\mu r}{\rho U_{0}}} \quad \theta=90^{\circ}
$$

Substituting this result into Equation (13) gives the film thickness profile along $\mathrm{AA}^{\prime}$ in the BLFZ,

$$
\frac{h}{d}=0 \cdot 125\left(\frac{d}{r}\right)+\frac{1.06}{\sqrt{R e_{j}}}\left(\frac{r}{d}\right)^{1 / 2}
$$

Equation (3) then gives the following relationship between film thickness, $h$, and average velocity $U_{a v}$ at radial position $r$ : 


$$
r h U_{a v}=\frac{Q}{2 \pi}=\frac{d^{2} U_{0}}{8}
$$

Solving Equations (24) and (25) yields the average velocity in the BLFZ:

$$
U_{a v}=\frac{U_{0}}{8 \frac{r}{d}\left(0 \cdot 125\left(\frac{d}{r}\right)+\frac{1.06}{\sqrt{R e_{j}}}\left(\frac{r}{d}\right)^{1 / 2}\right)}
$$

The radius where the boundary layer reaches the free surface, labelled $r_{\mathrm{b}}$, and after which liquid flows as a fully developed laminar film, is calculated by setting $h=\delta$ in Equation (13). An analytical result is available for $\theta=90^{\circ}$, viz.

$$
\frac{r_{b}}{d}=0.24 R e_{\mathrm{j}}^{1 / 3}
$$

The film thickness predicted by the above model is similar to that reported by previous workers: Liu et al. (1991), Watson (1964) and Azuma and Hoshino (1984 b) all reported expressions of the form $\frac{r_{b}}{d}=O\left(R e_{j}^{1 / 3}\right)$. Watson (1964) obtained $\frac{r_{b}}{d}=0.1578 R e_{j}^{1 / 3}$ for his similarity profile, whilst Liu et al. (1991) found that a cubic velocity profile gave $r_{\mathrm{b}} / d=$ $0.1773 R e_{j}^{1 / 3}$.

\section{Laminar film zone (LZ)}

Beyond $r_{\mathrm{b}}$ the liquid flows in a fully developed laminar film which eventually becomes turbulent (Azuma and Hoshino, 1984a). In the LZ, the velocity profile is again assumed to be described by the fourth order polynomial, for consistency with the BLFZ. In the LZ, however, $\eta=z / h$. Equation (15) becomes:

$$
u=U f(\eta) \quad 0<z<h
$$

Solving Equations (4), (22) and (28) with the initial condition that at $r=r_{b}, U_{b}=$ $\frac{1}{2} \sqrt{U_{0}^{2}-2 g r_{b} \cos \theta}$, yields the velocity profile in the laminar zone.

$$
\frac{d U_{a v}}{d r}=-\frac{5.7 \pi^{2} \mu U_{a v}^{2} r^{2}}{Q^{2} \rho}-\frac{0.714 g \cos \theta}{U_{a v}}
$$

This requires numerical integration except for $\theta=90^{\circ}$, where integrating Equation (4) with the boundary condition $U_{a v}=\frac{U_{0}}{2}$ at $r=r_{b}$ gives

$$
\left(\frac{Q}{\pi}\right)^{2}\left(\frac{1}{U_{a v}}-\frac{1}{U_{0} / 2}\right)=\frac{1.9 \mu}{\rho}\left(r^{3}-r_{b}^{3}\right) \quad \theta=90^{\circ}
$$

The average velocity in the film, $U_{a v}$, is 


$$
U_{a v}=\frac{U_{0}}{8 \frac{r}{d}\left(\frac{3.792}{R e_{j}}\left(\frac{r}{d}\right)^{2}+0.1975\left(\frac{d}{r}\right)\right)}
$$

This can be compared with the velocity profile obtained by Wilson et al. (2012), who assumed the film to have a fully developed parabolic velocity profile (i.e. a Nusselt film) where $r>d / 2$ :

$$
\frac{1}{U_{a v}}-\frac{1}{U_{o}}=\frac{10 \pi^{2} \mu}{3 \rho Q^{2}}\left[r^{3}-(d / 2)^{3}\right]
$$

This can be rearranged for comparison with Equation (31):

$$
U_{a v}(r)=\frac{U_{0}}{8 \frac{r}{d}\left(\frac{6.667}{R e_{j}}\left(\frac{r}{d}\right)^{2}+\frac{\left(3 R e_{j}-20\right)}{24 R e_{j}}\left(\frac{d}{r}\right)\right)} \approx \frac{U_{0}}{8 \frac{r}{d}\left(\frac{6.667}{R e_{j}}\left(\frac{r}{d}\right)^{2}+0.125\left(\frac{d}{r}\right)\right)}
$$

There is evident similarity between (31) and (33), providing some insight into the good agreement seen with the Wilson et al. model. It predicts similar trends.

Solving Equations (25) and (31) gives the film thickness in the LZ as

$$
\frac{h}{d}=\frac{3.792}{R e_{j}}\left(\frac{r}{d}\right)^{2}+0.1975\left(\frac{d}{r}\right) \quad \theta=90^{\circ}
$$

\section{Laminar to turbulent transition}

The laminar film eventually becomes turbulent at radius $r_{\mathrm{t}}$. Watson (1964) used the approximate result of Lin (1945) to determine the transition radius. Azuma and Hoshino (1984a) and Liu et al. (1991) found that Watson's turbulent model did not give good agreement with their experimental data and both groups presented alternative correlations for $r_{\mathrm{t}} / d$. Azuma and Hoshino set $r_{\mathrm{t}}$ to be the location where the film has granular waves of very small wave-length and they described these waves as sandpaper-like waves (see Azuma and Hoshino, 1984a). Liu et al. (1991) used two criteria: $(i)$ where the smooth laminar film lost its transparency and became a rough light scattering surface; and (ii) where there was a noticeable increase in the local Nusselt number (heat transfer coefficient). The latter results are compared with the new model in Supplementary Information S2. In our experiments, the transition is taken to be where smooth regularly spaced concentric waves become more chaotic. It will be shown that the correlations presented by Azuma and Hoshino (1984a) and Liu et al. (1991) give less good prediction of these features. 
Dou (2006) and Dou and Khoo (2010) proposed a mechanism for the laminar-turbulent transition based on flow instability. Duo proposed that the instability in a viscous flow can arise from the relative magnitude of the energy gradient in the transverse ( $z$ ) direction to that in the streamline $(r)$ direction arising from viscous friction. A large energy gradient in the transverse direction can potentially amplify a disturbance while that in the streamline direction can absorb these disturbances. From their analysis they concluded that, for pressure driven flows, an inflection in the velocity profile can give rise to turbulence. For shear driven flows, the condition for the transition is the existence of a zero velocity gradient in the velocity profile of the averaged flow (Dou and Khoo, 2010).

The flow field created by an impinging jet is a shear driven flow, where turbulence is promoted by surface waves, unlike the boundary layer on a flat plate where turbulence starts from the wall (Kline et al., 1967; Azuma and Hoshino 1984b; Dou and Khoo, 2010). In our experiments, the jet has a varicose shape owing to the Rayleigh-Plateau instability (Rayleigh, 1892), which on impingement creates surface waves on the film (Azuma and Hoshino 1984b). We postulate that the change in surface wave behaviour can be promoted by the existence of a singularity in the variation of energy with film thickness. This can amplify a disturbance and convert a smooth laminar flow into a more chaotic flow: such a location in the film can be a point of transition. We acknowledge that the hypothesis needs to be proven theoretically and experimentally.

The energy flux per unit width of the film is given by;

$$
E=\rho U_{a v}^{3} h+\rho g r U_{a v} h \cos \theta
$$

Substituting $U_{a v}$ from Equation (25) into (35)

$$
E=\rho\left(\frac{d^{2} U_{0}}{8 r h}\right)^{3} h+\frac{Q}{2 \pi} \rho g \cos \theta
$$

The variation with film thickness is

$$
\frac{d E}{d h}=\left(\frac{\partial E}{\partial r}\right) \frac{d r}{d h}+\left(\frac{\partial E}{\partial h}\right)
$$

Hence

$$
\frac{d E}{d h}=\frac{-3 d^{6} U_{0}^{3}}{256 r^{4} h^{2}}\left(\frac{d r}{d h}\right)-\frac{d^{6} U_{0}^{3}}{128 r^{3} h^{3}}
$$

When $\frac{d h}{d r}=0$ in the laminar region, there is a singularity in $\frac{d E}{d h}$ and this is associated with instability in the film. The condition for instability in film is then 


$$
\frac{d h}{d r}=0 \quad \text { (laminar region) }
$$

Solving Equations (34) and (39) yields the transition radius, $r_{\mathrm{t}}$, for the case where $\theta=90^{\circ}$ as

$$
\frac{r_{t}}{d}=0.2964\left(R e_{j}\right)^{1 / 3}
$$

\section{Turbulent region (TZ)}

At low jet Reynolds numbers, the film jump may occur before the laminar-turbulent transition. In cases where the film does become turbulent, the velocity profile is assumed to follow a $1 / 7^{\text {th }}$ power law and the wall shear stress, $\tau_{w}$, is calculated from the Blasius law (Azuma and Hoshin, 1984b; Liu et al., 1991; Schlichting, 1968).

$$
\frac{u}{U}=\eta^{1 / 7}
$$

Here $U$ is the surface velocity, and

$$
\frac{\tau_{w}}{\rho U^{2}}=0.0225\left(\frac{\mu}{\rho U h}\right)^{1 / 4}
$$

Ignoring the azimuthal component of velocity and integrating Equation (3) for a $1 / 7^{\text {th }}$ power law velocity profile (Equation 41) yields

$$
\frac{Q}{2 \pi}=\frac{7}{8} U r h=U_{a v} r h
$$

Substituting Equation (43) into Equation (42) gives

$$
\frac{\tau_{w}}{\rho U^{2}}=\frac{0.0366}{R e_{j}{ }^{1 / 4}}\left(\frac{r}{d}\right)^{1 / 4}
$$

Rearranging Equation (4) and integrating with respect to $\eta$ gives

$$
\frac{h U r}{r} \frac{d(U)}{d r} \int_{0}^{1} f^{2} d \eta=-\frac{\tau_{w}}{\rho}-g h \cos \theta
$$

Equation (45) can be further simplified to

$$
\frac{d U_{a v}}{d r}=-0.04706\left(\frac{2 \pi}{Q}\right) \frac{r^{5 / 4}}{\left(R e_{j} d\right)^{1 / 4}} U_{a v}^{2}-\frac{63}{64} \frac{g \cos \theta}{U_{a v}}
$$

At $r_{\mathrm{t}}$, continuity requires $U_{a v}$ (laminar) $=U_{a v}$ (turbulent) $=U_{t}$. The velocity distribution can then be calculated.

For $\theta=90^{\circ}$, Equation (46) can be solved with the initial condition, $U_{t}=0.422 U_{0}$ at $r=r_{t}=$ $0.2964 d\left(R e_{j}\right)^{1 / 3}$. The result is 


$$
U_{0} d^{2}\left(\frac{1}{U_{a v}}-\frac{1}{U_{t}}\right)=\left.\left(\frac{0.1673}{\left(R e_{j} d\right)^{1 / 4}}\right) r^{9 / 4}\right|_{r_{t}} ^{r}
$$

Rearranging Equation (47) yields

$$
U_{a v}=\frac{U_{0}}{\frac{0.167}{R e_{j}^{0.25}\left(\frac{r}{d}\right)^{9 / 4}+\left(2 \cdot 37-0.0108 R e_{j}^{1 / 2}\right)}}
$$

Substituting for $U_{a v}$ into Equation (43) gives the local film thickness:

$$
\frac{h}{d}=\frac{0.0209}{R e_{j}{ }^{1 / 4}}\left(\frac{r}{d}\right)^{5 / 4}+\left(0.296-0.001356 R e_{j}{ }^{1 / 2}\right)\left(\frac{d}{r}\right)
$$

For angles other than $90^{\circ}$, the velocity is obtained by numerical integration. Examples of the predicted velocity distribution, i.e. $U_{a v}$ versus $r$, are compared with new experimental measurements.

\subsection{Film jump}

In the Wilson et al. (2012) model the location of the film jump is determined by a balance between momentum and surface forces. They estimated the net surface tension force acting at the film jump as $\gamma(1-\cos \beta)$, where $\gamma$ is the surface tension and $\beta$ is the contact angle between the liquid and the wall. The $\cos \beta$ contribution comes from the solid-liquid contact line. Wang et al. (2013b) reported that at higher flow rates, the location of the film jump was independent of the nature of the wall material and could be calculated from their model by using an effective contact angle of $90^{\circ}$. The Wilson et al. model is revisited as the high speed video studies reported here elucidated some aspects of this behaviour.

High speed video of the initial stages of formation of the RFZ was used to obtain estimates of $U_{a v}$ for comparison with the above theory. An example of the early stages of a jet impinging perpendicularly on to a vertical wall is provided as Supplementary Video 1. Initially the liquid spreads out in an almost circular front. After a short period the liquid stops spreading outwards and the rope forms. The location of the film jump at $\theta=90^{\circ}$ does not change position with time: the rope falls beyond location $R_{\mathrm{j}}$ (see Figure 1 ). At $\theta=0^{\circ}$, directly above the point of impingement, once the rope forms gravity causes it to fall back over the spreading film. Therefore, in the upward direction, against gravity, the film jump is first found to occur at the outer edge of the rope and $R_{\mathrm{j}}$ is marked accordingly in Figure 1 . With a hydrophilic wall material the rope spreads outward and the film jump is then located 
somewhere inside the rope. At $\theta=90^{\circ}$ gravity does not cause the rope to move towards the film jump and the jump is located at the inner edge of the rope.

The initial stages of the formation of a film jump by an impinging jet on a vertical wall is similar to the departure radius observed with a water bell. Button et al. (2010) showed that when a water jet impinges vertically upwards on to the underside of a horizontal plate, the water spreads radially outwards in the form of a thin film. The spreading film leaves contact with the surface abruptly at the departure point and falls downwards, possibly giving a water bell. They reported that the departure point was independent of the nature of the surface material: however, the angle of departure was different for different materials.

Button et al. (2010) proposed that the departure point was where the outward momentum flux per unit width, $M$, was equal to the surface force:

$$
M=\int_{0}^{h} \rho u^{2} d z=\gamma
$$

The liquid film could not move further radially outwards. For the radial flow zone (RFZ) the film jump occurs when the momentum per unit circumferential width is equal to the surface tension. The liquid then flows circumferentially downwards in the rope. For a turbulent film at $\theta=90^{\circ}$, this gives

$$
M=\frac{\rho \frac{64}{632 \pi} U_{0}}{r\left\{\frac{0.167}{R e_{j}{ }^{0.25}}\left(\frac{r}{d}\right)^{9 / 4}+\left(2 \cdot 37-0.0108 R e_{j}{ }^{1 / 2}\right)\right\}}=\gamma
$$

The film jump is calculated by finding the value of $r$ that satisfies Equation (51), or its equivalent for the given value of $\theta$.

The observation that the sensitivity to the wall material disappears at higher flow rates is attributed to the shape of the rope. Experimental observations of the rope such as the photograph in Figure 3(a) suggests the rope shape shown in Figure $3(b) . R_{\mathrm{j}}$ is the radial location of the film jump at the given value of $\theta$ and $r_{\mathrm{H}}$ is the position where the rope height is largest. It is assumed that the radius of curvature of the rope at $r_{\mathrm{H}}$ is equal to the height of the rope there, labelled $H$. At this location the surface of the rope is continuous and differentiable, such that 


$$
\left.\frac{\partial H}{\partial r}\right|_{r=r_{H}}=0
$$

Applying a momentum balance between positions $R_{\mathrm{j}}$ and $r_{\mathrm{H}}$ gives

$$
M_{R_{j}}+\left.P h\right|_{R_{j}}=M_{r_{H}}+\left.P H\right|_{r_{H}}
$$

Here, $P$ is the gauge pressure. The $\left.P h\right|_{R_{j}}$ term can be ignored as the gauge pressure in the film is negligible (parallel streamlines) and the film thickness is small prior to the jump. The momentum in the radial direction at $r_{\mathrm{H}}$ is negligible as the net flow outwards inside the rope is zero. Hence,

$$
M_{R_{j}}=\left.P H\right|_{r_{H}}
$$

The gauge pressure inside the rope can be calculated by the Laplace equation, assuming that the radius of curvature at maximum height is $H$ :

$$
\left.P\right|_{r_{H}}=\gamma\left(\frac{1}{r_{H}}+\frac{1}{\mathrm{H}}\right)
$$

Substituting Equation (55) into Equation (54) and noting that $r_{\mathrm{H}} \approx R_{\mathrm{j}}$ yields

$$
M_{R_{j}}=\gamma\left(1+\frac{\mathrm{H}}{R_{j}}\right)
$$

At higher values of $R_{\mathrm{j}}$, or in the limit where $R_{\mathrm{j}} \gg H$, the term $\gamma\left(\frac{H}{R_{j}}\right)$ can be neglected and the momentum flux is approximately equal to the surface tension. The location of the film jump is then independent of the contact angle, in accordance with experimental observations.

Bush and Aristoff (2003) considered the effect of surface forces on the circular hydraulic jump. They calculated the contribution of the surface force to be given by $\gamma \Delta H / R_{\mathrm{j}}$, where $\Delta H$ is the difference in film heights across at hydraulic jump. The above result is consistent with this statement. Bush and Aristoff also reported that, for low flow rates (smaller $R_{\mathrm{j}}$ ), the surface force contribution is significant whereas for large values of $R_{\mathrm{j}}$, the contribution is very small. For the calculations in this work, at higher $R e_{\mathrm{j}}$, the surface force is a minor contribution.

The criterion for formation of the film jump at high flow rates is then $M_{R j}=\gamma$.

\subsection{Obliquely impinging jet}


The analysis is now extended to a liquid jet impinging obliquely on to a vertical surface. Immediately after impingement, the jet velocity, $U_{\mathrm{o}}$, remains the same; however, the amount of liquid flow per unit width varies with $\theta$. The azimuthal component of velocity is again omitted from the analysis. However, due to inclination of the jet, the amount of liquid flowing radially depends on $\theta$ : the distribution of flow for an obliquely impinging jet presented by Kate et al. (2007) was used by Wang et al. (2013b) and is used here. Oblique impingement of a circular jet gives an elliptical zone of impact (see Figure 4). Kate et al. showed that one of the foci of the ellipse, labelled S on Figure 4(b), acts as a source and the liquid moves radially away from this point. The radial distance from the source to the edge of the impingement zone, $r_{\mathrm{e}}$, subtended by angle $\theta$ is

$$
r_{e}=r_{0}\left(\frac{\sin \phi}{1+\cos \theta \cos \phi}\right)
$$

where $r_{\mathrm{o}}$ is the radius of the jet. Wang et al. (2013b) showed that the local average velocity in the RFZ is given by

$$
r h U_{a v}=\frac{1}{2} U_{0} r_{e}^{2} \sin \phi \quad r>r_{\mathrm{e}}
$$

Following the treatment leading to Equations (11), (12), (13) and (58), the film thickness in the BLFZ with oblique impingement is given by

$$
h=\frac{U_{0} r_{e}^{2} \sin \phi}{2 r U_{o}}+\left(1-C_{3}\right) \delta
$$

Solving Equations (9) and (59) yields the equation for the evolution of boundary layer thickness

$$
\frac{d\left(\delta^{2}\right)}{d r}=-\frac{2 \delta^{2}}{r}-\frac{2 \delta^{2} g \cos \theta}{U^{2}} \frac{\left(1-2 C_{1}+C_{3}\right)}{\left(C_{1}-C_{3}\right)}-\frac{2 C_{2} \mu}{\rho U\left(C_{1}-C_{3}\right)}+\frac{U_{0} r_{e}^{2} \sin \phi g \cos \theta \delta}{U_{o}\left(C_{1}-C_{3}\right) U^{2} r}
$$

Substituting Equation (59) into (58) gives

$$
U_{a v}=\frac{\frac{1}{2} U_{0} r_{e}^{2} \sin \phi}{r\left(\frac{U_{0} r_{e}^{2} \sin \phi}{2 r U_{o}}+\left(1-C_{3}\right) \delta\right)}
$$

where $h$ and $U_{a v}$ depend on both $\theta$ and $\phi$.

Similarly, in the LZ, Equation (29) takes the form

$$
\frac{d U_{a v}}{d r}=-\frac{5.7 \mu U_{a v}^{2} r^{2}}{\rho\left(U_{0} r_{e}^{2} \sin \phi\right)^{2}}-\frac{0.714 g \cos \theta}{U_{a v}}
$$

with, for $\theta=90^{\circ}$ 


$$
\left(U_{0} r_{e}^{2} \sin \phi\right)^{2}\left(\frac{1}{U}-\frac{1}{U_{0} / 2}\right)=\frac{1.9 \mu}{\rho}\left(r^{3}-r_{b}^{3}\right)
$$

For the TZ, Equation (46) becomes

$$
\frac{d U_{a v}}{d r}=-\frac{0.04706}{\left(\frac{1}{2} U_{0} r_{e}^{2} \sin \phi\right)\left(4 r_{e}^{2} \sin \phi\right)^{1 / 4}} \frac{d^{1 / 4} r^{5 / 4}}{\left(R e_{j}\right)^{1 / 4}} U_{a v}^{2}-\frac{63}{64} \frac{g \cos \theta}{U_{a v}}
$$

These results for the velocity and film thickness are used to find the location of the film jump, $R_{\mathrm{j}, \theta}$, which will vary with $\theta$. The predictions are compared with experimental data for $\theta=0^{\circ}$ and $90^{\circ}$ for a range of flow rates and angle of impingement $\phi$.

\section{Materials and methods}

\subsection{Experimental setup}

Two different apparatuses were used in this work. Figure 5 shows a schematic of the test rig used for the majority of the experiments. A detailed description of this system was given by Wilson et al. (2015). Liquid was pumped from an overhead supply tank through pressure, conductivity and temperature sensors before entering a flow control valve. The pressure upstream of the nozzle was measured to monitor the flow rate. These studies employed a solid stream nozzle of internal diameter $2.66 \mathrm{~mm}$ (Lechler GmbH, Type 544) to generate the jet. The nozzle mounting consisted of a $106 \mathrm{~mm}$ long section of i.d. $16 \mathrm{~mm}$ piping, including a pressure port connection, followed by a $50 \mathrm{~mm}$ section of i.d. $11 \mathrm{~mm}$ to which the nozzle was connected. This is more representative of industrial cleaning nozzle installations than the very long, smooth entry sections used by workers such as Azauma and Hoshino (1984a). The connections can introduce perturbations in the jet. The nozzle mounting allowed rotation so that the angle of impingement could be changed. The nozzle and target were located in a light-tight steel chamber, with illumination provided by externally mounted $1200 \mathrm{~W}$ halogen lamps. All the sensors, valves and pump were connected to a computer control and data collection system.

The nozzle was positioned $200 \mathrm{~mm}$ from the target, which was a $500 \times 500 \mathrm{~mm}$ and $5 \mathrm{~mm}$ thick plate made of Perspex ${ }^{\mathrm{TM}}$ or glass. A graticule tape was fixed on the target to provide in situ scale calibration. A white sheet of paper was mounted on the dry side of the plate in 
order to give better image resolution and contrast. The target was cleaned, wiped with ethanol, and allowed to dry before each experiment.

Deionised water at $23{ }^{\circ} \mathrm{C}$ was used as the test liquid. Initially liquid leaving the nozzle was prevented from reaching the target by a Perspex ${ }^{\mathrm{TM}}$ interrupter plate to allow the flow to reach steady state. After approximately 1 minute the interrupter plate was removed and the flow pattern created by the jet striking the surface was captured using a high speed camera (Phantom Miro M310) operating at 43000 frames per second. Supplementary Video 1 shows the jet striking the target and the formation of a circular front of liquid which grows and eventually forms a pattern similar to Figure 1. Images were processed using the NIH ImageJ software.

Although the nozzle was horizontal, gravity could cause the jet to droop a small amount as it traversed the $200 \mathrm{~mm}$ to the target. The angle of impingement was therefore slightly less. The effective angle of impingement was calculated and the values are reported in Table 1.

The second apparatus was described by Wang et al. (2013b) and was similar in layout to that in Figure 5 except that the walls were transparent rather than opaque. Tests employed deionized water at approximately $18^{\circ} \mathrm{C}$. Water was pumped from a $26 \mathrm{dm}^{3}$ capacity storage tank through a rotameter and a manual control valve before entering the nozzle. The nozzle was fabricated from brass with an orifice diameter of $2 \mathrm{~mm}$ : further details are given in Wang et al. (2013b). The nozzle could be rotated to give angles of impingement over the range $0<$ $\phi<180^{\circ}$. In these tests the nozzle was located between 40 to $80 \mathrm{~mm}$ from the target so that the jet remained coherent and did not droop. The target was either a transparent Perspex or glass plate. Transparent graticule tape was again mounted on the target to provide in-situ dimension calibration. The experiments performed in the second apparatus featured lower flow rates, with $Q=0.6$ to $2 \mathrm{dm}^{3} / \mathrm{min}$. An IP65 waterproof fluorescent tube lamp illuminated the target. Photographs and video were taken with a Nikon ${ }^{\mathrm{TM}}$ D 3300 camera and image processing was again performed using the ImageJ software.

\subsection{Image analysis and data processing}


Images were processed using NIH ImageJ software or Matlab® scripts. The radius of the growing RFZ was extracted and plotted against time in Origin ${ }^{\circledR}$. A polynomial was fitted to these data and differentiated to obtain an estimate of the average velocity, $U_{\mathrm{av}}$, in the fully developed RFZ at different values of $r$. For the measurement of the film jump, the images were processed in ImageJ and the location of the film jump was determined manually. 


\section{Results and Discussion}

The models presented in Section 2 provide estimates of film thickness, $h$, and average velocity $U_{a v}$. Both terms are needed to predict the location of the film jump, $R_{\mathrm{j}}$, using Equation (50). Film thicknesses were not measured in the experiments so the models are compared with results from the literature. Model predictions of $U_{a v}$ are compared with the values obtained from the initial growth of the RFZ.

\subsection{Film thickness}

Stevens and Webb (1993) measured the thickness of thin films of water as part of their study of the flow structure in the films with a free surface created by impinging liquid jets. They used laser-Doppler velocimetry (LDV) to determine the velocity profile in the film and calculated film thickness from conservation of volume. Figure $6(a)$ shows some of their experimental data alongside the model prediction, Equation (24). For this Reynolds number, of 53100 , Equation (27) predicts the boundary layer to reach the surface of the film at $r_{\mathrm{b}} \approx$ 9.01d, indicating that the data originate from the BLFZ. Similarly, Figure 6(b) compares the model predictions with data obtained at $R e_{\mathrm{j}}=37,000$ and 36,100 for jet diameters of $23 \mathrm{~mm}$ and $14 \mathrm{~mm}$, respectively. The small difference in $R e_{\mathrm{j}}$ does not give a significant difference in the predicted $h / d$ values. The predicted values of $r_{\mathrm{b}}$ are $8 d$ and 7.93d for $R e_{\mathrm{j}}=37,000$ and 36,100, respectively, again lying within the BLFZ. Error bars were not plotted on the experimental data. There is good agreement between the experimental results and the model in each case. It is noteworthy that there are no fitted parameters in the model.

\subsection{Transition radius, $r_{\mathrm{t}}$}

In the laminar zone, the liquid film thickness initially decreases and reaches a location where

it is minimum. At this location $\frac{d E}{d h}$ shows a singularity and we hypothesize that the laminar film becomes unsteady and turbulent effects are expected to dominate. Equation (39) gives the location of the transition for the general case, and Equation (40) for $\theta=90^{\circ}$. Azuma and Hoshino (1984b) presented the following correlation for the radius of a transition based on their visual observations 


$$
\frac{r_{t}}{d}=730\left(R e_{j}\right)^{-0.315}
$$

Whereas Liu et al. (1991) reported two correlations, one based on visual observation,

$$
\frac{r_{t}}{d}=1200\left(R e_{j}\right)^{-0.422}
$$

and one based on heat transfer data, viz.

$$
\frac{r_{t}}{d}=28600\left(R e_{j}\right)^{-0.68}
$$

Figure 7(a) compares the present model, for $\theta=90^{\circ}$, and the above correlations. The prediction for $r_{\mathrm{t}} / d$ differs noticeably at lower $R e_{\mathrm{j}}$. Equations (65) and (66) show a similar trend but the former is consistently larger by about 15 units. All three (Equations (65-67) predict a decrease in $r_{t} / d$ with increasing Reynolds number, whereas the current model, Equation (40), predicts an increasing trend. Equations (40), (66) and (67) predict similar values of $r_{\mathrm{t}} / d$, in the range 9-15, for $R e_{\mathrm{j}}>70000$.

Figure 7(b) compares the present model predictions, Equation (40), for $r_{\mathrm{t}} / d$ with our experimental measurements of the location where the smooth concentric waves started to break. Two sets of predictions are reported as the jet exhibited a varicose structure, where surface tension causes the diameter to vary periodically, before impinging. The maximum and minimum jet diameters were extracted from photographs and used in the prediction. For a $2.667 \mathrm{~mm}$ nozzle, the maximum and minimum measured jet diameters were 2.86 and 2.66 $\mathrm{mm}$. The experimental data lie between the two sets of model predictions corresponding to minimum and maximum jet diameters and shows good agreement with the model.

The model also gave good agreement with transitions reported by other workers. Liu et al. (1991) reported measurement of local heat transfer coefficient (and Nusselt number) for liquid jets impinging perpendicularly on a heated plate. Their plots of local Nusselt number vs $r / d$ show a generally decreasing trend but with a local maximum at $r / d$ around 6-10. They attributed this local maximum to the onset of turbulence in the RFZ and quantified it as Equation (67). Their data are compared with the current model in Supplementary Information $\mathrm{S} 2$. At higher $R e_{\mathrm{j}}$ the model gives a reasonably accurate prediction of this local maximum.

Rao et al. (1964) measured the local rate of mass transfer for a water jet impinging on a horizontal plate coated with a soluble layer. They reported the local Sherwood number for a 
range of Reynolds numbers and jet diameters. At low $R e_{\mathrm{j}}$, careful inspection of local Sherwood number vs $r / d$ profiles shows a deviation from the general decreasing trend, similar to that reported by Liu et al. for heat transfer. The location of the deviation is close the transition predicted by the present model (data not presented).

In the derivation of the criterion for the transition from laminar to turbulent film behaviour, it is postulated, following the work of Azuma et al. (1984a) that the transition is accompanied by the loss of regularity in surface waves. The photographs in Figure 8 show regular concentric waves in the RFZ: further from the point of impingement these are less coherent. The prediction for the transition (Equation 40) is plotted on the image and shows good agreement with the observed feature. By comparison, the Liu et al. (1991) correlation, Equation (66), consistently overpredicts the transition.

\subsection{Velocity profile in RFZ}

\subsubsection{Perpendicular impingement $\left(\phi=90^{\circ}\right)$}

Measurements of the velocity profile in these films are complicated by the presence of surface waves. Aouad et al. (2016) measured the surface velocity in the RFZ for jets impinging perpendicularly on vertical walls using particle image velocimetry. They used a dye to limit measurements to the surface region alone. The measured surface velocities were noticeably higher than those predicted by the Wilson et al. (2012) model and this was attributed to the presence of surface waves. In the present study the average film velocity was estimated from the initial evolution of the flow pattern until a steady rope was formed.

Figure 9 compares the average velocity profile for $\theta=90^{\circ}$ obtained from video analysis for flow rates ranging from $1.95-4.01 \mathrm{dm}^{3} / \min \left(R e_{\mathrm{j}}=15,500\right.$ to 32,000$)$ with the predictions of the new model as well as that of Wang et al. (2013b). The latter is based on that of Wilson et al. (2012) and includes the correction for an obliquely impinging jet and gravity. There are no adjustable parameters in either model and the agreement is good in most cases, until the approach to the film jump itself. The agreement is less good for $R e_{\mathrm{j}}=32,000$ but the general trend is captured.

Comparing the two models, the Wang et al. (2013b) predictions show consistently less good agreement. It underpredicts the average velocity at larger radial position but the difference is 
less than a factor of 2. In several cases the two models bracket the measured velocity. This indicates that the Wilson et al. (2012) model provides a reasonable engineering estimate of the average film velocity. It is sufficiently accurate for engineering estimates of phenomena determined by the film such as the location of the film jump and cleaning for $\phi=90^{\circ}$ (see Wilson et al. 2014; Glover et al., 2016).

\subsubsection{Oblique impinging jet $\left(\phi=120^{\circ}\right)$}

The obliquely impinging jet presents a stiffer test for the model as the distribution of liquid as well as the film development are tested. Figure 10 presents results for different flow rates for a challenging case, where the jet impinges in an upward direction $\left(\phi=120^{\circ}\right)$ : Wang et al. (2013b) showed that the widest part of the RFZ was then located above the impingement point. The Figure presents pairs of plots for each case studied; for $\theta=0^{\circ}$ (above the impingement point, where flow is against gravity) and $\theta=180^{\circ}$ (downstream of the impingement point). The effective angle of impingement, reported in Table 1, was used in the calculations. For this value of $\phi, r_{\mathrm{e}}$ is largest at $\theta=0^{\circ}$ and smallest for $\theta=180^{\circ}$ : for a given flow rate the maximum liquid flux occurs at $\theta=0^{\circ}$ and the smallest at $\theta=180^{\circ}$.

There is a noticeable kink in the new model, at the laminar to turbulent transition. This is not evident in the estimated average velocity data, but the model nevertheless gives a reasonably good estimate of the experimental data. These are, we believe, the first time that measurements of the average velocity have been made for obliquely impinging jets.

\subsection{Location of the film jump}

\subsubsection{Horizontal jets impinging perpendicularly}

The film jump is located where the outward flow of momentum is countered by surface tension. Above the plane $\mathrm{AA}^{\prime}$ in Figure 1, the liquid collects to form the rope and drains under gravity around the RFZ. Below plane AA', initially, a film jump was clearly observed on wall materials like Perspex (Supplementary Video 3). However, owing to gravitational acceleration in the downward direction, the film jump eventually gives way to a transition to a draining film in steady state. Therefore, the film jump was only observed above plane AA'. Above $\mathrm{AA}^{\prime}$ the film jump is not circular: this is partly due to gravity retarding the upwards flow but also due to the dynamics of the rope. Supplementary Videos 1 and 2 show that at small values of $\theta$ the film jump and RFZ formed is initially almost circular: over time, the 
rope grows in size and spreads downwards into the RFZ. At larger values of $\theta$, such as $90^{\circ}$, the rope spreads outwards, the extent to which it spreads being determined by the nature of the surface, i.e. a hydrophilic wall material favouring a wider rope. This observation indicates that the predictions of the location of the film jump should be compared with the outer dimension of the rope at $\theta=0^{\circ}$ rather than the inner limit.

Figure 11 compares the measured values of $R_{\mathrm{j}}$ for $\theta=90^{\circ}\left(\phi=90^{\circ}\right)$ for flow rates between 0.6-4 dm $3 / \min \left(R e_{\mathrm{j}}=6,300\right.$ to 32,000) for Equations (51) and the Wilson et al., (2012) model. In all cases the jets are turbulent. Following the discussion in Section 2.2, the latter model uses an effective contact angle of $90^{\circ}$. Figure 11(a) presents data collected with a $2 \mathrm{~mm}$ nozzle on Perspex on the second apparatus, while those in Figure 11(b) were obtained with tests using a $2.667 \mathrm{~mm}$ nozzle on Perspex ${ }^{\mathrm{TM}}$ on the first apparatus. The Wilson et al. (2012) model underpredicts $R_{\mathrm{j}}$ but gives a reasonable estimate, which is consistent with the predictions of average velocity in Figure 10.

Figure $11(b)$ compares results obtained at higher flow rates $\left(1.95-4.01 \mathrm{dm}^{3} / \mathrm{min}\right)$ for two different wall materials, Perspex ${ }^{\mathrm{TM}}$ and glass. The model gives very good agreement with the data for Perspex but consistently underpredicts the observations on glass, by 2-4 $\mathrm{mm}$. On glass, which is hydrophilic, the liquid spreads on the surface and there is more noticeable, random, variation in the film jump location. Button et al. (2010) reported a similar, small variation in the departure radius of water bells on glass.

Comparing the models indicates that the new model provides a more reliable prediction for the location of the film jump than those based on the Wilson et al. (2012) approach.

\subsubsection{Oblique impingement on a vertical surface}

Predicting the location of the film jump for an obliquely impinging jet is, like the average velocity profile, a sterner test of the model. Figure 12 compares the experimental measurements of film jump location for $\theta=0^{\circ}$ and $90^{\circ}$ and a range flow rates, 1.95-4.01 $\mathrm{dm}^{3} /$ min and angles of impingement $\phi=120^{\circ}$ and $100^{\circ}$. For $\phi=120^{\circ}$, a large portion of the flow travels upward and gives rise to a very unstable rope: the measurements of $R_{\mathrm{j}}$ were therefore made when the rope was first formed. The difference in flow rates gives rise to $R_{\mathrm{j}}$ $\left(\theta=0^{\circ}\right)$ being almost twice $R_{\mathrm{j}}\left(\theta=90^{\circ}\right)$. The locus for the Wang et al. (2013b) model shows that it tended to overpredict $R_{\mathrm{j}}\left(\theta=0^{\circ}\right)$. 
In contrast, the new model gives good agreement with the experimental data for both directions. Similarly good agreement was obtained for other values of $\theta$ (data not reported), indicating that it provides a acceptably accurate description of the flow behaviour within the jet for angles of impingement in the range $30<\phi<120^{\circ}$

\subsection{Every man's fluid mechanics}

The oblique impingement of a liquid jet on a vertical wall is familiar to many in the context of male urination in modern toilets. The model is applied here in a somewhat jocular vein to test its predictive capacity for this everyday phenomenon.

A typical adult male urethral orifice is elliptical with dimensions $6 \mathrm{~mm} \times 0.5 \mathrm{~mm}$ and delivers $1-1.4 \mathrm{dm}^{3} /$ min urine (Drake et al., 2007). Hodgson and Smith (2014) conducted a short study mimicking male urination against a vertical well. They established the typical angle of impingement during urination to lie between $30^{\circ}-60^{\circ}$ and measured $R_{\mathrm{j}, 90}$ and $R_{\mathrm{j}, 0}$ for a $2 \mathrm{~mm}$ diameter nozzle at angle of impingements $40^{\circ}, 50^{\circ}$ and $60^{\circ}$ against a vertical Perspex ${ }^{\mathrm{TM}}$ sheet. They studied saline solution $\left(16 \mathrm{~g} \mathrm{dm}^{-3}\right.$, mimicking urine) and water at $37^{\circ} \mathrm{C}$ at flow rates of $1,1.2$ and $1.4 \mathrm{dm}^{3} / \mathrm{min}$. The density, viscosity and surface tension of saline solution at $37^{\circ} \mathrm{C}$ were similar to that of water (given in parentheses), as 1009 (993) $\mathrm{kg} \mathrm{m}^{-3}, 0.71$ (0.696) $\mathrm{mPa}$ s and $0.0704(0.0701) \mathrm{N} \mathrm{m}^{-1}$ respectively (Hodgson and Smith., 2014). 
The film jump measurements are compared with the present model, Equation (50), in Figure 13. The horizontal distance between the wall and the nozzle was $105 \mathrm{~mm}$ and some drooping of the jet due to gravity was encountered. The effective angle of impingement was estimated and the values are given in Table 2. The model gives a reasonable prediction of their film jump measurements.

The model does not account for splashback, which can cause social problems. Hurd et al. (2013) characterized the splash back form male urination and recommended that practitioners employ a low angle of impingement, $\phi$, and a short distance of travel so that the jet impinges as a coherent jet. If the jet is not close enough, jet break-up due to the Rayleigh-Plateau instability results in more splash back. The model confirms that a small value of $\phi$ will cause the bulk of the liquid to flow downwards. We leave it to the reader to explain to practitioners that they should stand close to the wall in order to generate coherent jets.

\subsection{Wall shear stress in the RFZ}

The shear stress imposed by the flow in the film is an important parameter in applications such as heat transfer and in cleaning (where the soiling layer is eroded by the flow). Yeckel and Middelman studied the removal of an oil layer by a water jet impinging perpendicularly downwards on to a horizontal plate (giving a hydraulic jump). The shear stress imposed on the oil layer by the water film caused it to thin over time. They presented a two zone model for the flow in the RFZ based on Watson's analysis, with a turbulent film throughout. They obtained the following results for $\tau_{\mathrm{w}}$ :

$$
\begin{array}{ll}
\tau_{w}=0.0397 \frac{\rho U_{o}^{2}}{2} \operatorname{Re}_{j}^{-0.2}\left(\frac{r}{r_{o}}\right)^{-0.2} & r<r_{+} \\
\tau_{w}=30.3 \frac{\rho U_{o}^{2}}{2}\left(\frac{r}{r_{o}}\right)^{0.25} \frac{\operatorname{Re}_{j}^{0.25}}{\left[\left(\frac{r}{r_{o}}\right)^{2.25}+27.24 \operatorname{Re}_{j}^{0.25}\right]^{2}} & r>r_{+}
\end{array}
$$

where $r^{+}$is the radius at which the turbulent boundary layer reaches the free surface, given by 


$$
\frac{r_{+}}{r_{o}}=1.84 \mathrm{Re}_{j}^{1 / 9}
$$

Yeckel and Middelman used the jet radius as the length scale in the jet Reynolds number: Equations (68-70) are written in terms of $R e_{\mathrm{j}}$ as defined above, i.e. with jet diameter as the characteristic length scale. The wall shear stress distribution predicted by Equations (68-69) for an example case are compared with those obtained from the velocity distributions given by Liu et al. (1991), Wilson et al. (2012), and the current work (based on that by Azuma and Hoshino (1984a,b)), in Figure 14. The wall shear stress is evaluated in each zone using

$$
\tau_{\mathrm{w}}=\left.\mu \frac{\partial \mathrm{u}}{\partial \mathrm{z}}\right|_{\mathrm{z}=0}
$$

(i) This work

In the BLFZ, differentiating Equation (15) yields

$$
\tau_{w}=\left.\mu \frac{U}{\delta} f^{\prime}(\eta)\right|_{0}
$$

For $\theta=90^{\circ}$, this gives

$$
\tau_{w}=\mu \frac{U_{0}}{2.12 \sqrt{\frac{\mu r}{\rho U_{0}}}}
$$

In the laminar zone, the shear stress is given by

$$
\tau_{w}=\left.\mu \frac{U}{h} f^{\prime}(\eta)\right|_{0}
$$

For $\theta=90^{\circ}$

$$
\tau_{w}=\mu \frac{U_{0}}{4 r\left(\frac{3.792}{R e_{j}}\left(\frac{r}{d}\right)^{2}+0.1975\left(\frac{d}{r}\right)\right)^{2}}
$$

In the turbulent zone, the wall shear stress is calculated from the Blasius law, giving

$$
\tau_{w}=\frac{0.0478 \rho}{R e_{j}{ }^{1 / 4}}\left(\frac{U_{0}}{\frac{0.167}{R e_{j}{ }^{0.25}}\left(\frac{r}{d}\right)^{9 / 4}+\left(2 \cdot 37-0.0108 R e_{j}{ }^{1 / 2}\right)}\right)^{2}\left(\frac{r}{d}\right)^{1 / 4}
$$


(ii) Liu et al. (1991)

Applying a similar treatment to this flow profile gives

Boundary layer

$$
\tau_{w}=\mu \frac{1.5 U_{0}}{2.679 \sqrt{\frac{r d}{R e_{j}}}}
$$

Laminar zone

$$
\tau_{w}=\mu \frac{0.3 U_{0}}{r\left(\frac{5.147}{R e_{j}}\left(\frac{r}{d}\right)^{2}+0.1713\left(\frac{d}{r}\right)\right)^{2}}
$$

(iii) Wilson et al. (2012)

Likewise, the approximation of a Nusselt film yields the result

$$
\tau_{w}=\mu \frac{3 U_{0}}{8 r\left(\frac{6.667}{R e_{j}}\left(\frac{r}{d}\right)^{2}+\frac{\left(3 R e_{j}-20\right)}{24 R e_{j}}\left(\frac{d}{r}\right)\right)^{2}}
$$

The shear stress distribution predicted by Equation (79) differs noticeably from the other profiles until larger values of $r / d$, when the assumption of a Nusselt film is a more reasonable description of the flow in the film. The maximum in $\tau_{\mathrm{w}}$ at $r / d \sim 5$ is not present in the other cases as this location lies in the initial region where the boundary layer is still developing: boundary layer development is not considered in the Wilson et al. (2012) model. The trends in Figure 14 indicate that the Wilson et al. model is unlikely to give reliable descriptions of erosive cleaning driven by surface shear stress. Figures 9 and 10 show that the Wilson et al. model does give reasonable estimates for $U$, and consequently the momentum in the film, which is the key parameter in the model for adhesive removal (i.e. peeling) presented by the same group (Wilson et al., 2014).

The Figure shows that the current model predicts a similar distribution in $\tau_{\mathrm{w}}$ to the Liu et al. model. The Yeckel and Middelman model predicts a noticeably larger shear stress than the other detailed models for $r / d<7$ : this is because their wall shear stress calculation is for a turbulent film, using the Blasius law, and is higher in this region than the shear stress in a laminar film. Thereafter the trends are similar, with the prediction from the current work, Equation (75), lying between the other two results. This pattern was observed in other cases considered. 


\section{Conclusions}

The flow pattern created by the impingement of a turbulent water jet on a vertical wall was investigated using glass and Perspex ${ }^{\mathrm{TM}}$ walls. The thin film in the radial flow zone was modelled as three regions and the governing equations were solved for each to find the velocity field and the film thickness. The film thickness measurements reported by Stevens and Webb, (1993) were used to guide the model: this in turn gave predictions of the average velocity in the film which were compared with new sets of data generated using high-speed video. The analysis yielded predictions for the transition to turbulent flow based on the location where the laminar liquid film can amplify a disturbance and become chaotic. Furthermore, the criterion for the location of the film jump proposed by Wilson et al. (2012) was revised and yielded a result which could explain the insensitivity of this feature to the nature of the wall material at high flow rates.

The postulation for the criterion for transition from a laminar film to a turbulent one is based by work of Duo and co-workers, and relates the transition to the point where there is a minimum in film thickness. In the experiments the transition was assigned to be the distance where concentric transverse surface waves (arising from the jet being varicose due to a Plateau-Rayleigh instability) were replaced by more chaotic structures. This hypothesis requires more rigorous theoretical analysis and is a topic for further study.

The measurements of the average velocity in the film were obtained using a new technique. It avoids the complications experienced using velocimetry where the influence of waves is significant (see Aouad et al., 2015). It is relatively robust, independent of liquid optical properties and can be readily applied to different wall materials, angles of inclination and flow rates.

The model gave good agreement with both the historical and new data, for average velocity and location of the film jump. The predictions for obliquely impinging jets were noticeably superior to the model of Wang et al., (2013b) and Wilson et al., (2012). This will allow the rate of cleaning by such jets, using the model of Wilson et al. (2014), to be predicted with greater confidence. 


\section{Acknowledgements}

Funding for RKB from the Commonwealth Scholarship Commission is gratefully acknowledged, as are helpful discussions with Prof. J.F. Davidson, Dr. David Scott and Mr. Ole Mathis Magens. Author RKB performed the tests on the apparatus in Figure 5 as part of a visit funded by the TU Dresden Graduate Academy: related insightful discussions with Mr. Hannes Köhler and Prof. J-P Majschak are also gratefully acknowledged. 


\section{Appendix}

Ignoring the effect of drag forces, the estimated $\theta$ component of velocity arising from gravitational acceleration is given by

$$
\frac{d v_{\theta}}{d t}=g \sin \theta
$$

The rate of radial spread of liquid is

$$
\frac{d r}{d t}=U_{a v}
$$

So one can write

$$
d t=\frac{d r}{U_{a v}}
$$

Substituting Equation A1-3 into A1-1, rearranging and integrating yields

$$
\int_{0}^{v_{\theta}} d v^{\prime}{ }_{\theta}=\int_{0}^{r} g \sin \theta \frac{d r \prime}{U_{a v}}
$$

To estimate the magnitude of $v_{\theta}$, the average radial velocity in the RFZ is estimated using the result from Wilson et al. (2012)

$$
U_{a v}(r)=\frac{U_{0}}{8 \frac{r}{d}\left(\frac{6.667}{R e_{j}}\left(\frac{r}{d}\right)^{2}+\frac{\left(3 R e_{j}-20\right)}{24 R e_{j}}\left(\frac{d}{r}\right)\right)}
$$

Substituting Equation A1-5 into Equation A1-4 yields

$$
v_{\theta}=\int_{r_{0}}^{r} \frac{8 g \sin \theta r^{\prime}}{U_{o} d}\left(\frac{6.667}{R e_{j}}\left(\frac{r^{\prime}}{d}\right)^{2}+\frac{\left(3 R e_{j}-20\right)}{24 R e_{j}}\left(\frac{d}{r^{\prime}}\right)\right) d r^{\prime}
$$

Integrating Equation A1-6 gives

$$
v_{\theta}=\frac{g \sin \theta}{R e_{j} U_{o}}\left(\frac{40}{3 d^{3}}\left(r^{4}-r_{o}^{4}\right)+\frac{\left(3 R e_{j}-20\right)}{3}\left(r-r_{o}\right)\right)
$$

This is plotted in Figure A1 for two flow rates, representative of the range employed in these tests, for $r<R_{\mathrm{j}, 90}$. Over much of the RFZ $U_{\mathrm{av}}$ in the radial direction is significantly larger than $v_{\theta}$. The exception is where $r$ approaches $R_{\mathrm{j}, 90}$ and the film jump is approached. For the bulk of the film omitting $v_{\theta}$ from the analysis is a reasonable assumption. 


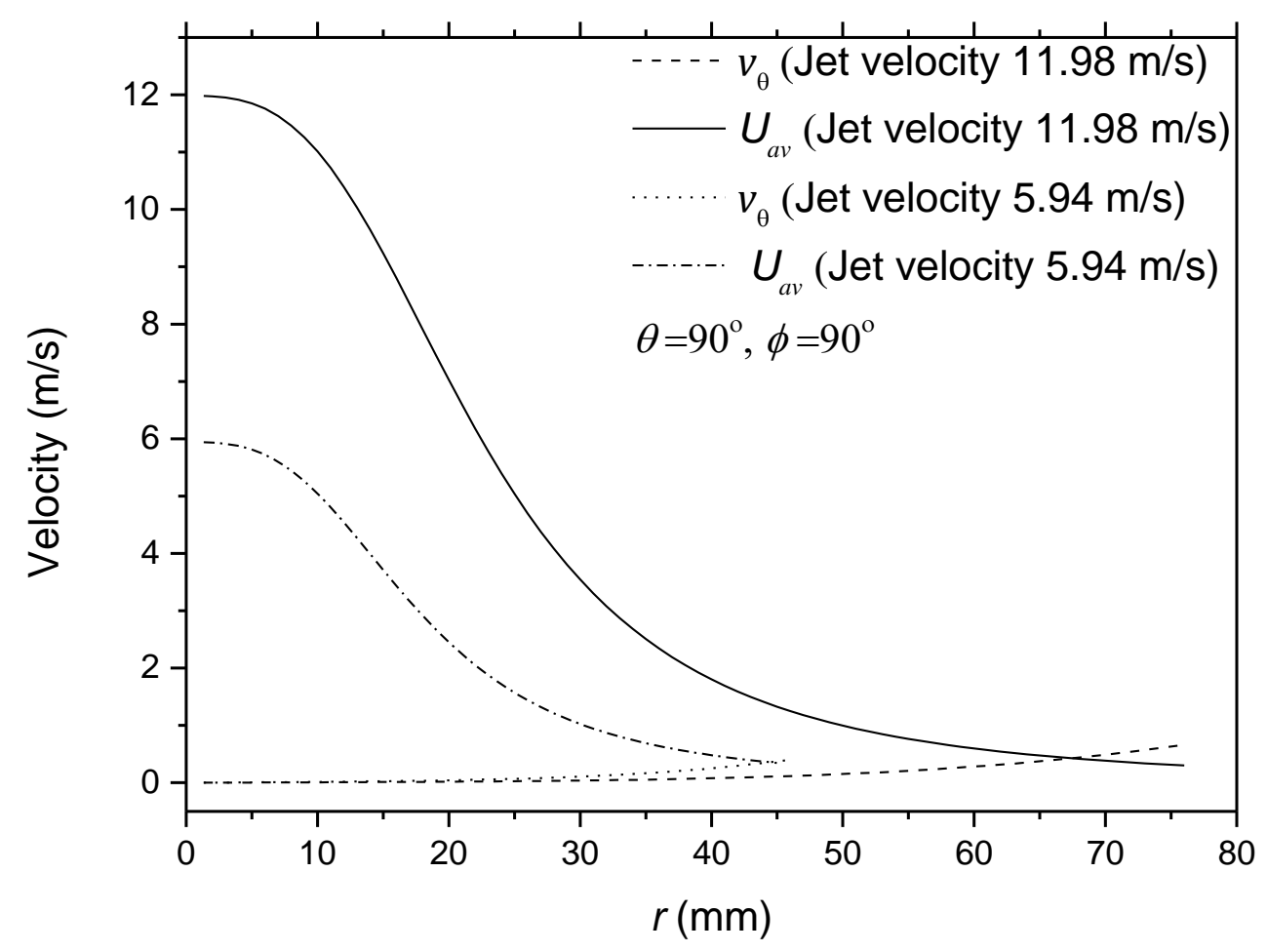

Figure A1 comparison of radial and azimuthal components of velocity in the RFZ for $\theta=90^{\circ}$, for flow rates of 4.01 and $1.99 \mathrm{dm}^{3} / \mathrm{min}$. The angle of impingement, $\phi$, is $90^{\circ}$. 


\section{Nomenclature}

\section{Roman}

$a_{\mathrm{i}}$

Coefficients in Equation (16)

$C_{1} \quad$ Constant $\left(\int_{0}^{1} f^{2}(\eta) d \eta=C_{1}\right)$, Equation (9)

$C_{2} \quad$ Constant $\left(\left.f^{\prime}(\eta)\right|_{0}=C_{2}\right)$, Equation (9)

$C_{3} \quad$ Constant $\left(\int_{0}^{1} f(\eta) d \eta=C_{3}\right)$, Equation (13)

$d$

Jet diameter

$\mathrm{m}$

$d_{\mathrm{N}}$

Nozzle diameter

$\mathrm{m}$

E

Mechanical energy flux per unit width

$\mathrm{kg} \mathrm{m} \mathrm{s}^{-3}$

$g$

Gravitational acceleration

$\mathrm{m} \mathrm{s}^{-2}$

$h$

Film thickness

$\mathrm{m}$

$H$

Maximum height of the rope, at $r=r_{\mathrm{H}}$

$\mathrm{m}$

$\dot{m} \quad$ Mass flow rate of liquid in the jet

$\mathrm{kg} \mathrm{s}^{-1}$

M

Momentum per unit width (Equation 50)

$\mathrm{kg} \mathrm{s}^{-2}$

$N u_{\mathrm{d}} \quad$ Nusselt number

$P$

Pressure

$\mathrm{Pa}$

$Q \quad$ Volumetric flow rate

$\mathrm{m}^{3} \mathrm{~s}^{-1}$

Radial co-ordinate

$\mathrm{m}$

Jet radius $\left(r_{\mathrm{o}}=d / 2\right)$

$\mathrm{m}$

Radius where boundary layer reaches the surface

$\mathrm{m}$

Radius where boundary layer is fully developed, (Equation 70) $\mathrm{m}$

$r_{\mathrm{t}}$

Transition radius from laminar to turbulent flow

$\mathrm{m}$

$r_{e}$

Radial distance from the focus to the boundary of the

$\mathrm{m}$

elliptical projection of a normal jet (Equation 57)

$R_{\mathrm{j}} \quad$ Radius at film jump

$\mathrm{m}$

$r_{H}$

Radius at the maximum height of the rope

$\mathrm{m}$

$R e_{\mathrm{j}} \quad$ Jet Reynolds number based on nozzle diameter

$t$

Time

$$
\text { s }
$$

$u \quad$ Radial velocity

$\mathrm{m} \mathrm{s}^{-1}$

$U_{\mathrm{o}}$

Jet velocity

$\mathrm{m} \mathrm{s}^{-1}$ 


$\begin{array}{lll}U_{a v} & \begin{array}{l}\text { Average radial velocity in liquid film } \\ U_{\mathrm{t}}\end{array} & \begin{array}{l}\text { Average radial velocity in liquid film at laminar to turbulent } \\ \text { transition radius }\end{array} \\ \begin{array}{ll}\text { Radial velocity at the liquid film surface } \\ v\end{array} & \mathrm{~m} \mathrm{~s}^{-1} \\ v_{\theta} & \text { Velocity normal to the surface } & \mathrm{m} \mathrm{s}^{-1} \\ z & \text { Velocity in azimuthal direction } & \mathrm{m} \mathrm{s}^{-1} \\ & \text { Distance normal to the surface } & \mathrm{m}\end{array}$

\section{Greek}

$\begin{array}{lll}\beta & \text { Contact angle } & \circ \\ \gamma & \text { Surface tension } & \mathrm{N} \mathrm{m}^{-1} \\ \delta & \text { Boundary layer thickness } & \mathrm{m} \\ \eta & \text { Dimensionless distance normal to the wall } & - \\ \theta & \text { Azimuthal angle } & \circ \\ \mu & \text { Dynamic viscosity } & \mathrm{Pa} \mathrm{s} \\ \rho & \text { Density } & \mathrm{kg} \mathrm{m}^{-3} \\ \phi & \text { Angle of inclination of the jet to the vertical } & \circ \\ \tau_{w} & \text { Wall shear stress } & \mathrm{Pa}\end{array}$

\section{Acronyms}

BLFZ Boundary layer flow zone

LZ Laminar zone

TZ Turbulent zone

RFZ Radial flow zone 


\section{References :}

Aouad, W., Landel, J.R., Dalziel, S.B., Davidson, J.F., Wilson, D.I., 2016. Particle image velocimetry and modelling of horizontal coherent liquid jets impinging on and draining down a vertical wall. Experimental Thermal and Fluid Science, 74, 429-443

Arakeri, J.H., Rao, K.A., 2013. On radial film flow on a horizontal surface and the circular hydraulic jump. Journal of the Indian Institute of Science, 76, 73.

Azuma, T., Hoshino, T., 1984a. The radial flow of a thin liquid film: 1st Report, Laminar to turbulent transition . Bulletin of JSME 27, 2739-2746.

Azuma, T., Hoshino, T., 1984b. The radial flow of a thin liquid film: 3rd Report, Velocity Profile. Bulletin of JSME 27, 2755-2762.

Bohr, T., Dimon, P., Putkaradze, V., 1993. Shallow-water approach to the circular hydraulic jump. Journal of Fluid Mechanics 254, 635-648.

Blyth, M.G., Pozrikidis, C., 2005. Stagnation-point flow against a liquid film on a plane wall. Acta Mechanica 180, 203-219.

Burfoot, D., Middleton, K., 2009. Effects of operating conditions of high pressure washing on the removal of biofilms from stainless steel surfaces. Journal of Food Engineering 90, 350-357.

Bush, J.W., Aristoff, J.M., 2003. The influence of surface tension on the circular hydraulic jump. Journal of Fluid Mechanics 489, 229-238.

Button, E.C., Davidson, J.F., Jameson, G.J., Sader, J.E., 2010. Water bells formed on the underside of a horizontal plate. Part 2. Theory. Journal of Fluid Mechanics 649, 4568.

Dou, H.-S., 2006. Mechanism of flow instability and transition to turbulence. International Journal of Non-Linear Mechanics 41, 512-517.

Dou, H.-S., Khoo, B.C., 2010. Criteria of turbulent transition in parallel flows. Modern Physics Letters B 24, 1437-1440.

Drake, R., Vogl, A.W., Mitchell, A.W., Tibbitts, R. and Richardson, P., 2007.Gray's atlas of anatomy. 1st ed. Churchill-Livingstone, UK.

Glover, H.W., Brass, T., Bhagat, R.K., Davidson, J.F., Pratt, L., Wilson, D.I., 2016. Cleaning of complex soil layers on vertical walls by fixed and moving impinging liquid jets. Journal of Food Engineering, 178, 95-109.

Gordeev, S., Groeschel, F., Stieglitz, R., 2015. Numerical analysis of high-speed lithium jet flow under vacuum conditions. Fusion Engineering \& Design, in press. doi:10.1016/j.fusengdes.2015.10.033

Hodgson P. J., and Smith, M.J. 2014, Flow patterns and cleaning behaviour of impinging liquid jets. CET IIB Research Project Report, Department of Chemical Engineering and Biotechnology, University of Cambridge.

Hurd, R., Hacking, K. \& Truscott, T.T., 2013,Urinal dynamics, 66th Annual Meeting of the American Physical Society Division of Fluid Dynamics, Pittsburg, PA.

Kate, R.P., Das, P.K., Chakraborty, S., 2007. Hydraulic jumps due to oblique impingement of circular liquid jets on a flat horizontal surface. Journal of Fluid Mechanics 573, 247 263.

Kline, S.J., Reynolds, W.C., Schraub, F.A., Runstadler, P.W., 1967. The structure of turbulent boundary layers. Journal of Fluid Mechanics 30, 741-773.

Landreth, C.C. and Adrian, R.J., 1990. Impingement of a low Reynolds number turbulent circular jet onto a flat plate at normal incidence. Experiments in Fluids, 9(1-2), 74-84.

Leach, S.J. and Walker, G.L. 1966, The application of high speed liquid jets to cutting, Phil. Trans. Royal Soc. London Series A, Mathematical and Physical Sciences, Vol 260, No 1110, pp. 295-310.

Lienhard, J., 1995. Liquid jet impingement. Annual Review of Heat Transfer 6, 199-270. 
Lienhard, J.H., 2006. Heat transfer by impingement of circular free-surface liquid jets, Proceedings of 18th National and 7th ISHMT-ASME Heat and Mass Transfer Conference, Guwahati, India.

Lin, C.C., 1945. On the stability of two-dimensional parallel flows Part II-stability in an inviscid fluid. Quart. Appl. Math. 3, 218-234.

Liu, X., Lienhard, J.H., Lombara, J.S., 1991. Convective heat transfer by impingement of circular liquid jets. Journal of Heat Transfer 113, 571-582.

Morison, K.R. and Thorpe, R.J., 2002. Liquid distribution from cleaning-in-place sprayballs. Food and Bioproducts Processing, 80(4), 270-275.

Nusselt, W., 1916. Die Oberflachenkondesation des Wasserdamffes the surface condensation of water. Zetrschr. Ver. Deutch. Ing., 60, 541-546.

Pérez-Mohedano, R., Letzelter, N., Amador, C., Van der Roest, C.T., Bakalis, S., 2015. Positron Emission Particle Tracking (PEPT) for the analysis of water motion in a domestic dishwasher. Chemical Engineering Journal 259, 724-736.

Rao, V., Trass, O., 1964. Mass transfer from a flat surface to an impinging turbulent jet. The Canadian Journal of Chemical Engineering 42, 95-99.

Rayleigh, L., 1892. XVI. On the instability of a cylinder of viscous liquid under capillary force. The London, Edinburgh, and Dublin Philosophical Magazine and Journal of Science 34, 145-154.

Schlichting, H., 1968. Boundary-Layer Theory. Springer Science \& Business Media.

Stevens, J., Webb, B.W., 1993. Measurements of flow structure in the radial layer of impinging free-surface liquid jets. International Journal of Heat and Mass Transfer 36, 3751-3758.

Wang, T., Davidson, J.F., Wilson, D.I., 2013a. Effect of surfactant on flow patterns and draining films created by a static horizontal liquid jet impinging on a vertical surface at low flow rates. Chemical Engineering Science 88, 79-94.

Wang, T., Faria, D., Stevens, L.J., Tan, J.S.C., Davidson, J.F., Wilson, D.I., 2013b. Flow patterns and draining films created by horizontal and inclined coherent water jets impinging on vertical walls. Chemical Engineering Science 102, 585-601.

Wang, T., Davidson, J.F., Wilson, D.I., 2015. Flow patterns and cleaning behaviour of horizontal liquid jets impinging on angled walls. Food and Bioproducts Processing 93, 333-342.

Watson, E.J., 1964. The radial spread of a liquid jet over a horizontal plane. Journal of Fluid Mechanics 20, 481-499.

Wilson, D.I, Atkinson, P., Köhler, H., Mauermann, M., Stoye, H., Suddaby, K., Wang, T., Davidson, J.F., Majschak, J.-P., 2014. Cleaning of soft-solid soil layers on vertical and horizontal surfaces by stationary coherent impinging liquid jets. Chemical Engineering Science 109, 183-196.

Wilson, D.I, Köhler, H., Cai, L., Majschak, J.-P., Davidson, J.F, 2015. Cleaning of a model food soil from horizontal plates by a moving vertical water jet. Chemical Engineering Science 123, 450-459.

Wilson, D.I, Le, B.L., Dao, H.D.A., Lai, K.Y., Morison, K.R., Davidson, J.F., 2012. Surface flow and drainage films created by horizontal impinging liquid jets. Chemical Engineering Science 68, 449-460.

Yeckel, A, and Middleman, S. (1987). Removal of a viscous film from a rigid plane surface by an impinging liquid jet. Chemical Engineering Communications, 50, 165-167. 


\section{Tables}

Table 1 Effective angle of impingement for jets inclined at $\phi=90^{\circ}$ and $120^{\circ}$ as a result of travel across the $200 \mathrm{~mm}$ distance between the nozzle and target.

\begin{tabular}{ccc}
\hline Flow rate & \multicolumn{2}{c}{ Effective angle of impingement } \\
$\mathrm{dm}^{3} / \mathrm{min}$ & $\phi=120^{\circ}$ & $\phi=90^{\circ}$ \\
\hline 1.95 & 116.7 & 85.5 \\
2.52 & 118.0 & 87.4 \\
3.05 & 118.6 & 88.2 \\
3.57 & 119.0 & 88.7 \\
4.01 & 119.2 & 88.9 \\
\hline
\end{tabular}

Table 2 Effective angle of impingement for jets with notional angle of impingement $\phi$ following travel across the $105 \mathrm{~mm}$ distance between the nozzle and target.

\begin{tabular}{|c|c|c|c|}
\hline \multirow{2}{*}{$\begin{array}{l}\text { Flow rate } \\
\mathrm{dm}^{3} / \mathrm{min}\end{array}$} & \multicolumn{3}{|c|}{ Effective angle of impingement } \\
\hline & $\phi=40^{\circ}$ & $\phi=50^{\circ}$ & $\phi=60^{\circ}$ \\
\hline 1 & 42 & 51.4 & 61 \\
\hline 1.2 & 42 & 51.4 & 61 \\
\hline 1.4 & 42 & 51.4 & 61 \\
\hline
\end{tabular}




\section{Figure captions}

Figure 1 Schematic of flow pattern formed by a jet impinging normally on a vertical wall. $(a)$ side view through section $\mathrm{BB}^{\prime}(b)$ front view. $\mathrm{O}$ is the point of impingement, $U_{\mathrm{o}}$ is the jet velocity and $d$ is the jet diameter.

Figure 2 Cross-section through the radial flow zone and the film jump at $\theta=90^{\circ}$. The different flow zones are: (1) stagnation region; (2) boundary layer formation, shown by dashed line; (3) boundary layer reaches the surface and laminar flow zone starts; (4) laminar to turbulent transition; (5) film jump; (6) rope. $O$ is the point of impingment.

Figure 3 Flow in the RFZ and rope: (a) photograph on glass wall $\left(R e_{\mathrm{j}}=22200, \phi=90^{\circ}\right.$, nozzle diameter $2 \mathrm{~mm}$ ) (b) Schematic, not to scale, showing a cross section of the rope.

Figure 4 Schematics of a liquid jet impinging obliquely on a vertical wall. ( $a$ ) side and end views, showing non-circular RFZ; $(b)$ end view, showing elliptical impact region.

Figure 5 Schematic of the test rig used to study the initial stages of RFZ formation. Labels:

$\mathrm{P}_{1}, \mathrm{P}_{2}$ - pressure sensors; $\mathrm{T}$ - temperature sensor; $\mathrm{K}$ - conductivity sensor; FCV flow control valve.

Figure 6 Comparison of liquid film thickness in the RFZ measured by Stevens and Webb (1993) with the model for the boundary layer zone, Equation (24). (a) $R e_{\mathrm{j}}=53100, d$ $=10.9 \mathrm{~mm} ;(b)$ Two different jets were used, with $(i) R e_{\mathrm{j}}=37000, d=23 \mathrm{~mm}$ and (ii) $R e_{\mathrm{j}}=36100, d=14 \mathrm{~mm}$. The test liquid was water at room temperature.

Figure 7 Location of the laminar-turbulent transition, $r_{\mathrm{t}}$. (a) Comparison of this work with existing correlations: $(b)$ Experimental observations of the critical radius for wave breakup alongside the model predictions, Equation (40). The error bars show the standard error of the measured transition radius.

Figure 8 Photographs of the RFZ and draining films obtained with perpendicular impinging jets, $d=2.667 \mathrm{~mm}$ and (a) $R e_{\mathrm{j}}=15500$ and (b) $R e_{\mathrm{j}}=28000$. Dashed line - this work $(d=2.87 \mathrm{~mm})$, solid line - this work $(d=2.67 \mathrm{~mm})$, Equation $(40)$; dotted line - Liu et al. (1991) correlation, Equation (66). 
Figure 9 Comparison of experimental estimate of the average velocity $U_{a v}$ obtained from video analysis of the formation of the RFZ with the present model. Vertical glass plate, nozzle diameter $2.667 \mathrm{~mm}, Q=$ (a) $1.95 \mathrm{dm}^{3} / \mathrm{min}$, (b) $2.49 \mathrm{dm}^{3} / \mathrm{min}$, (c) 3.01 $\mathrm{dm}^{3} / \min ,(d) 3.51 \mathrm{dm}^{3} / \mathrm{min}$, and (e) $4.01 \mathrm{dm}^{3} / \mathrm{min}$.

Figure 10 Comparison of estimated average velocity with model predictions for an obliquely impinging water jet $\left(\phi=120^{\circ}\right)$ on a vertical Perspex ${ }^{\mathrm{TM}}$ plate, nozzle diameter 2.667 $\mathrm{mm}$, for $Q=$ (a) $1.99 \mathrm{dm}^{3} / \mathrm{min}$, (b) $2.52 \mathrm{dm}^{3} / \mathrm{min}$; (c) $3.05 \mathrm{dm}^{3} / \mathrm{min}$; (d) $4.01 \mathrm{dm}^{3} / \mathrm{min}$; (1) $\theta=0^{\circ}$, (2) $\theta=180^{\circ}$.

Figure 11 Location of film jump for a jet impinging perpendicularly on a vertical surface at $\theta$ $=90^{\circ}$. Comparison of measured values and the model, Equation (51) for (a) $0.6 \leq Q \leq$ $2 \mathrm{dm}^{3} / \min , d=2 \mathrm{~mm}$, and (b) $1.95 \leq Q \leq 4.01 \mathrm{dm}^{3} / \mathrm{min}, d=2.667 \mathrm{~mm}$.

Figure 12 Location of the film jump for a jet impinging obliquely on a vertical surface. Comparison of measured and predicted values of $R_{\mathrm{j}}$ at $\theta=0^{\circ}$ and $90^{\circ}$. Predictions are presented for the present model and that of Wang et al. (2013b). $d=2.667 \mathrm{~mm}, Q=$ $1.95-4.01 \mathrm{dm}^{3} / \mathrm{min}$ : angle of impingement $\phi=100^{\circ}$ and $120^{\circ}$.

Figure 13 Every man's fluid mechanics: location of film jump at $\theta=90^{\circ}$ and $0^{\circ}$ for $Q=1,1.2$ and $1.4 \mathrm{dm}^{3} / \mathrm{min}$ and notional angles of impingement $40^{\circ}, 50^{\circ}$ and $60^{\circ}$ (Equation 50). Data taken from Hodgson and Smith (2014). Liquid temperature $37^{\circ} \mathrm{C}, d=2 \mathrm{~mm}$ and $R e_{\mathrm{j}}=10$ 600, 12 700, and 14 900. Loci show predictions from this work.

Figure 14 Comparison of the estimated wall shear stress distributions for a jet with $d=2 \mathrm{~mm}$, $R e_{\mathrm{j}}=21000$, flow rate $2 \mathrm{dm}^{3} \mathrm{~min}^{-1}, \theta=90^{\circ}$. 


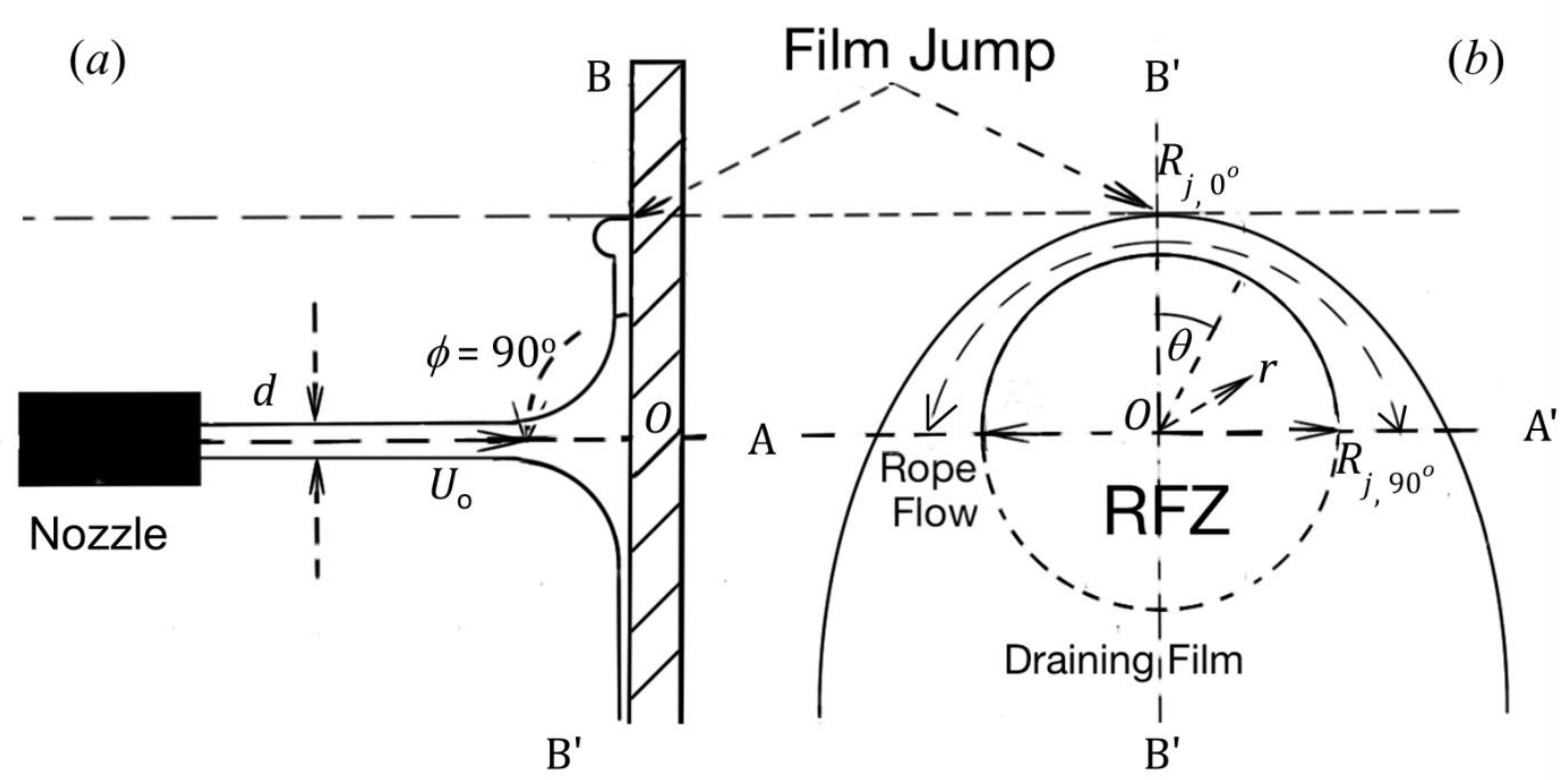

Figure 1 Schematic of flow pattern formed by a jet impinging normally on a vertical wall. $(a)$ side view through section $\mathrm{BB}^{\prime}(b)$ front view. $\mathrm{O}$ is the point of impingement, $U_{\mathrm{o}}$ is the jet velocity and $d$ is the jet diameter. 


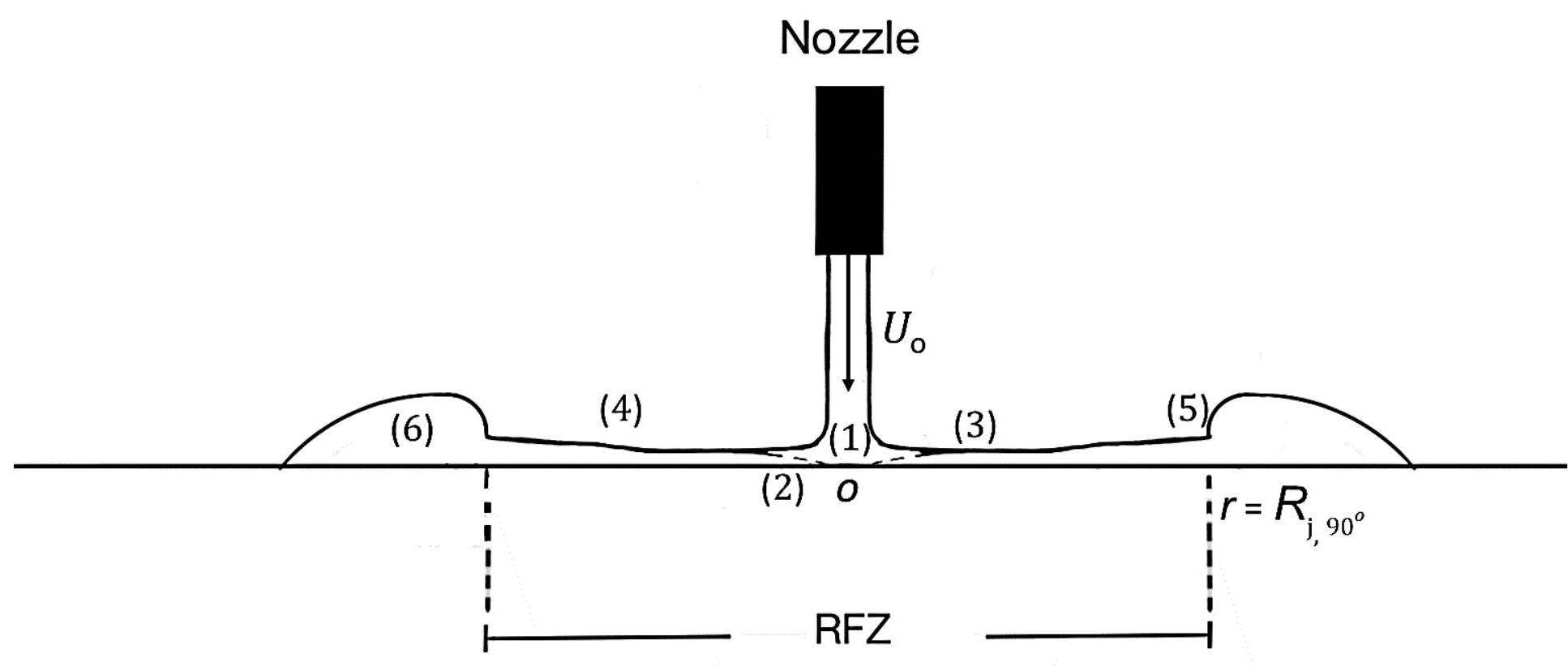

Figure 2 Cross-section through the radial flow zone and the film jump at $\theta=90^{\circ}$. The different flow zones are: (1) stagnation region; (2) boundary layer formation, shown by dashed line; (3) boundary layer reaches the surface and laminar flow zone starts; (4) laminar to turbulent transition; (5) film jump; (6) rope. $O$ is the point of impingment. 


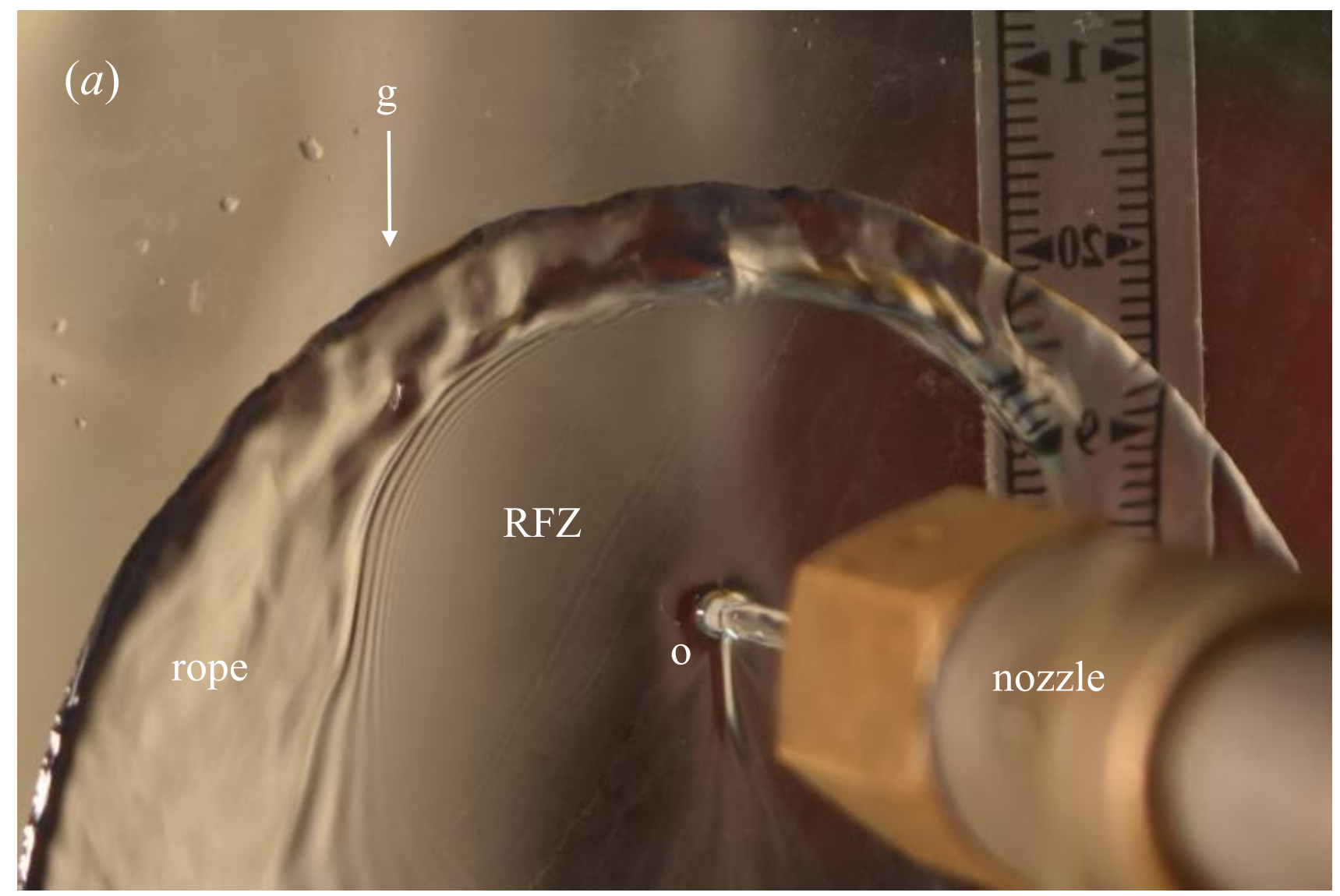


(b)

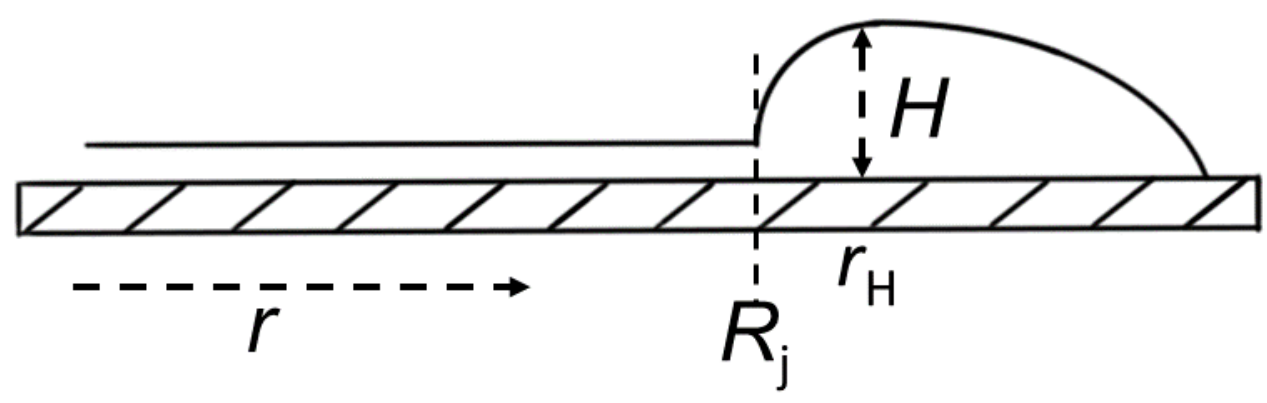

Figure 3 Flow in the RFZ and rope: (a) photograph on glass wall $\left(R e_{\mathrm{j}}=22200, \phi=90^{\circ}\right.$, nozzle diameter $2 \mathrm{~mm}$ ) (b) Schematic, not to scale, showing a cross section of the rope.

(a)

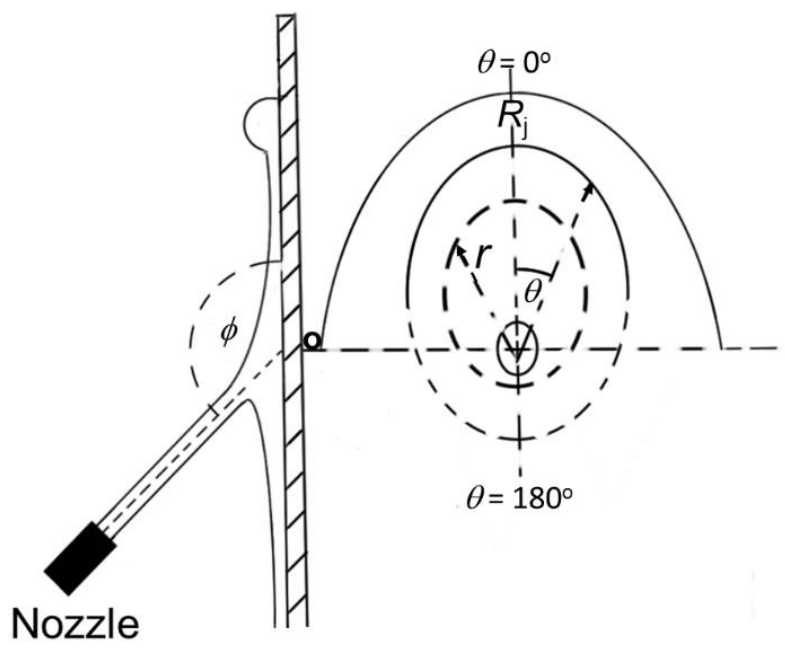




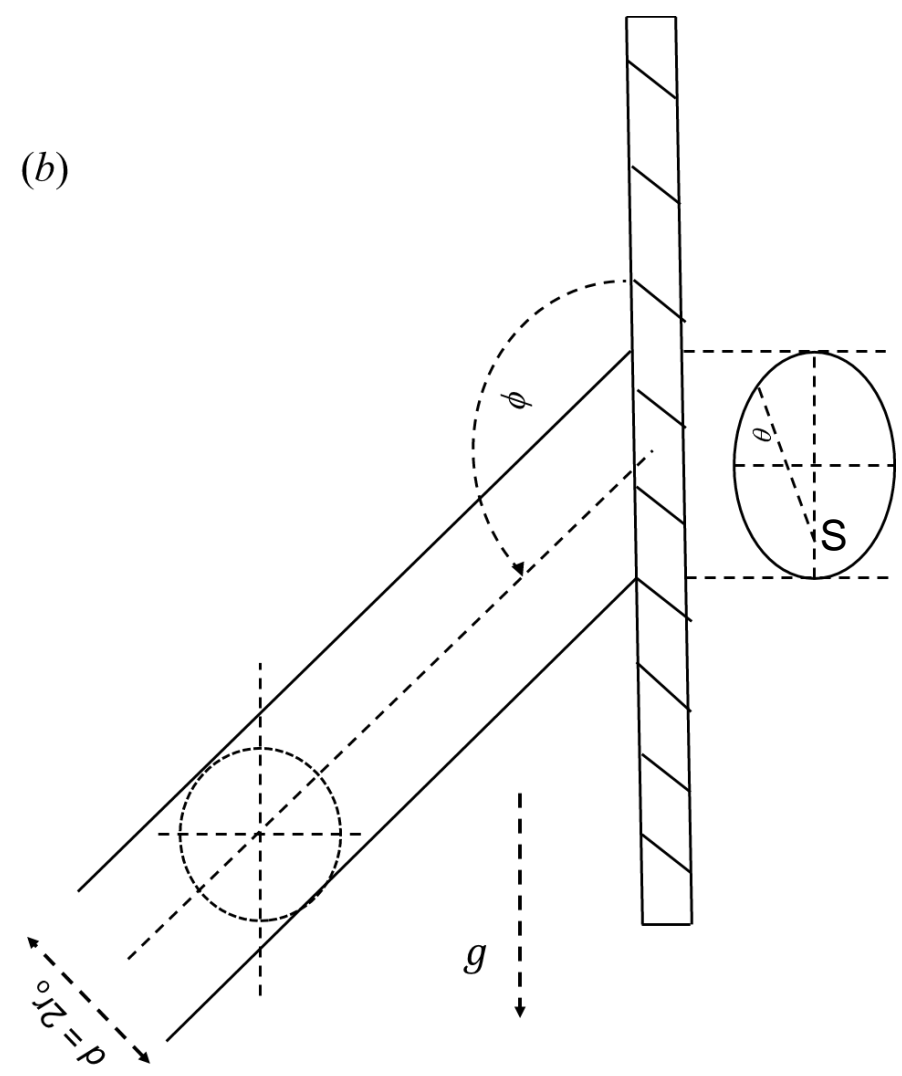

Figure 4 Schematics of a liquid jet impinging obliquely on a vertical wall. (a) side and end views, showing non-circular RFZ; $(b)$ end view, showing elliptical impact region. 


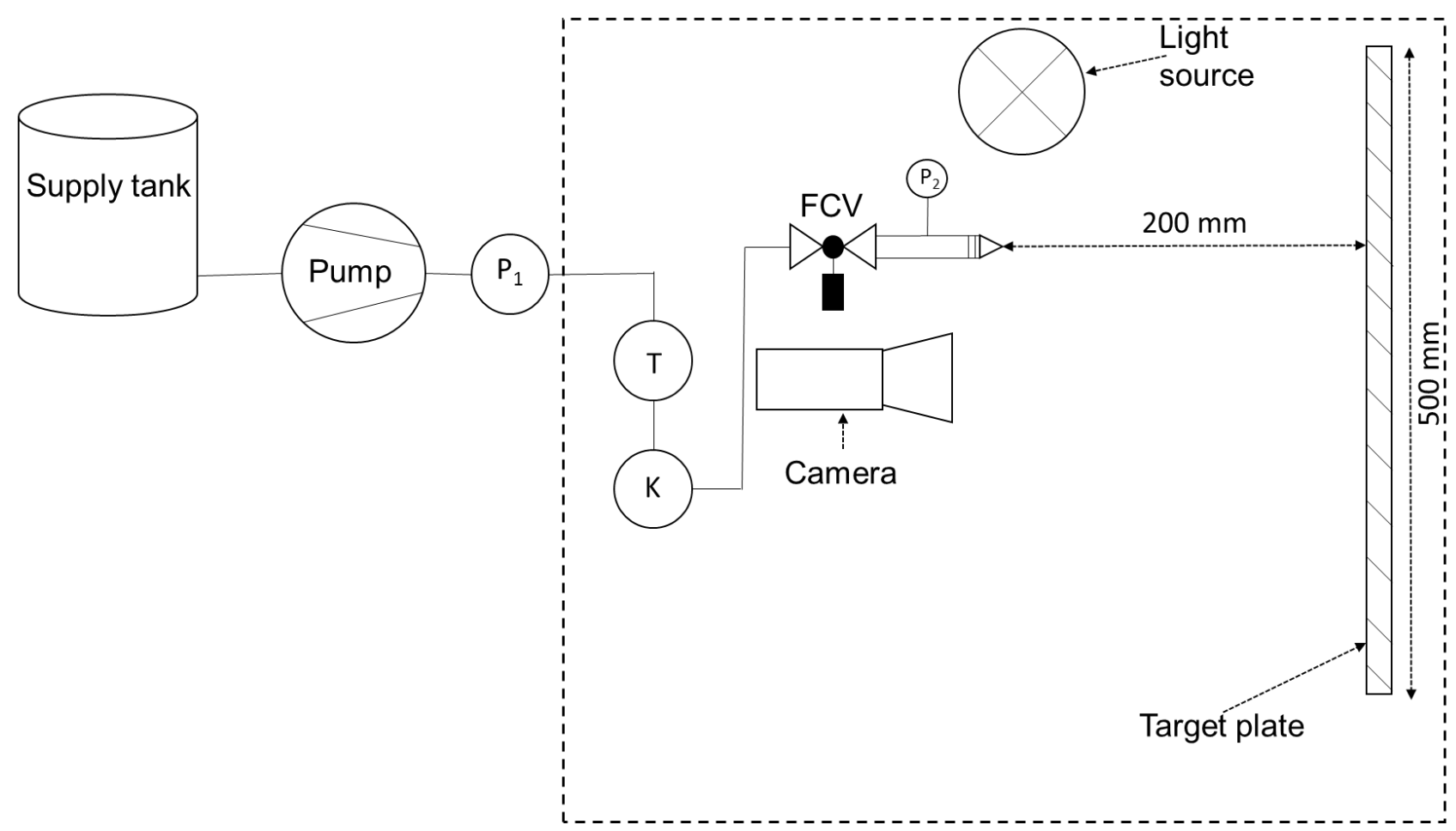

Figure 5 Schematic of the test rig used to study the initial stages of RFZ formation. Labels:

$\mathrm{P}_{1}, \mathrm{P}_{2}$ - pressure sensors; $\mathrm{T}$ - temperature sensor; $\mathrm{K}$ - conductivity sensor; FCV flow control valve. 

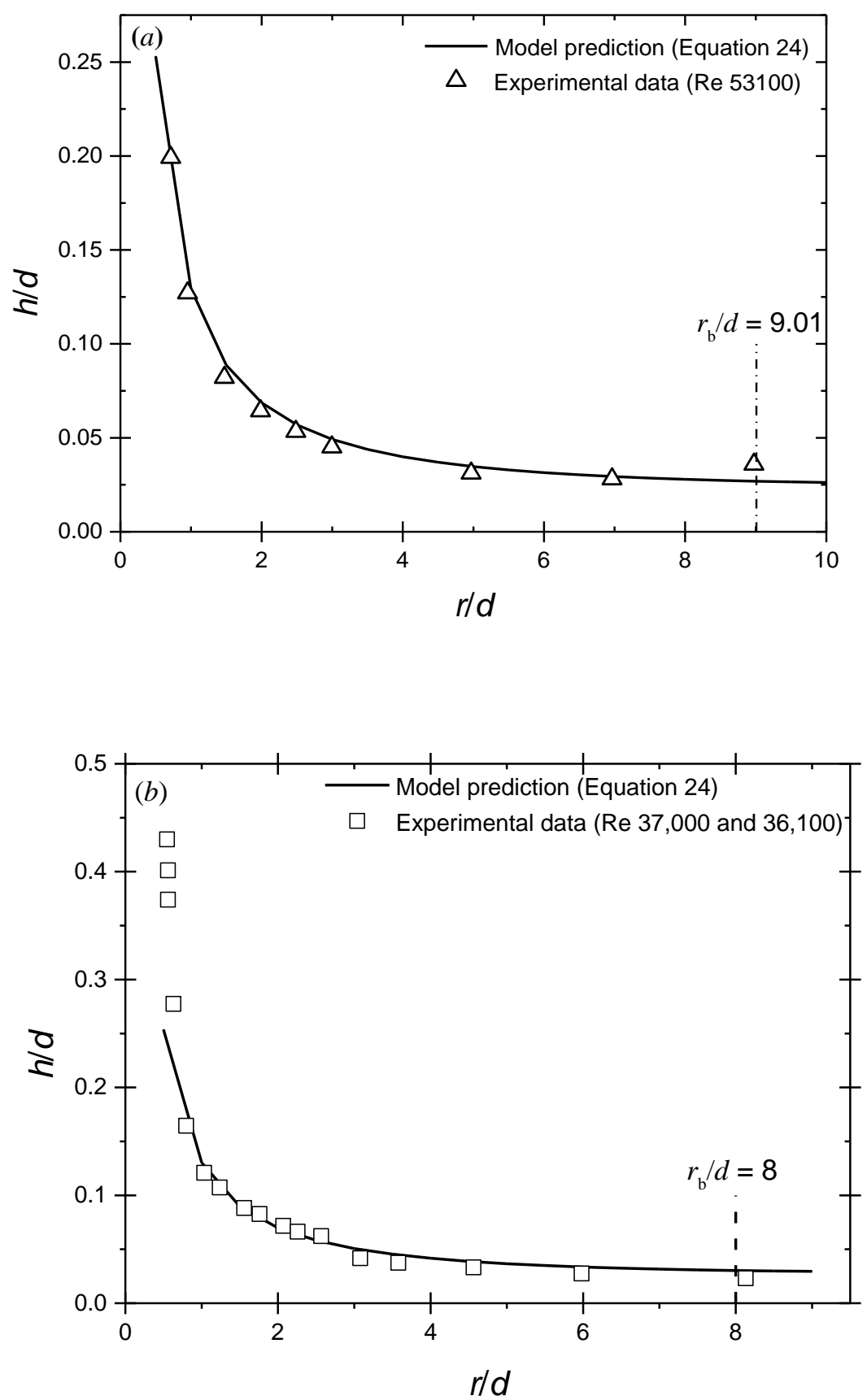

Figure 6 Comparison of liquid film thickness in the RFZ measured by Stevens and Webb (1993) with the model for the boundary layer zone, Equation (24). (a) $R e_{\mathrm{j}}=53100, d$ $=10.9 \mathrm{~mm} ;(b)$ Two different jets were used, with $(i) R e_{\mathrm{j}}=37000, d=23 \mathrm{~mm}$ and (ii) $R e_{\mathrm{j}}=36100, d=14 \mathrm{~mm}$. The test liquid was water at room temperature. 


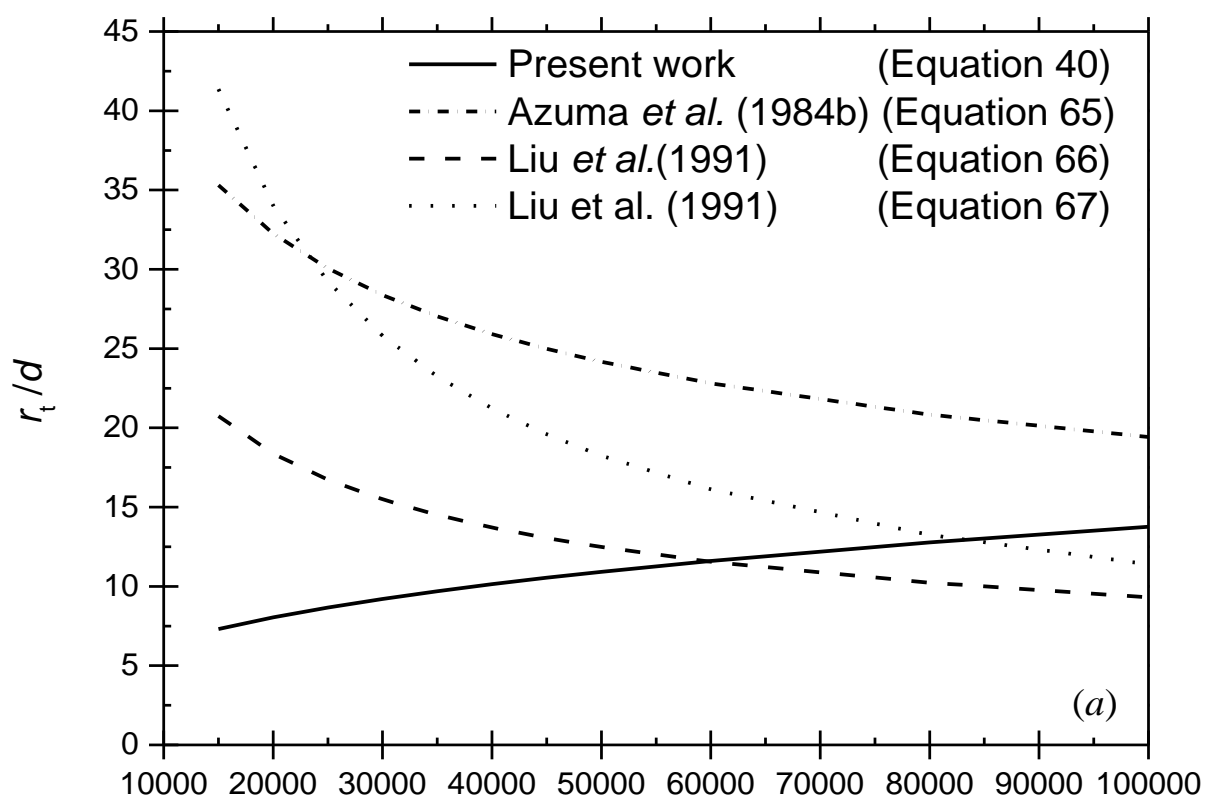

$R e_{\mathrm{j}}$

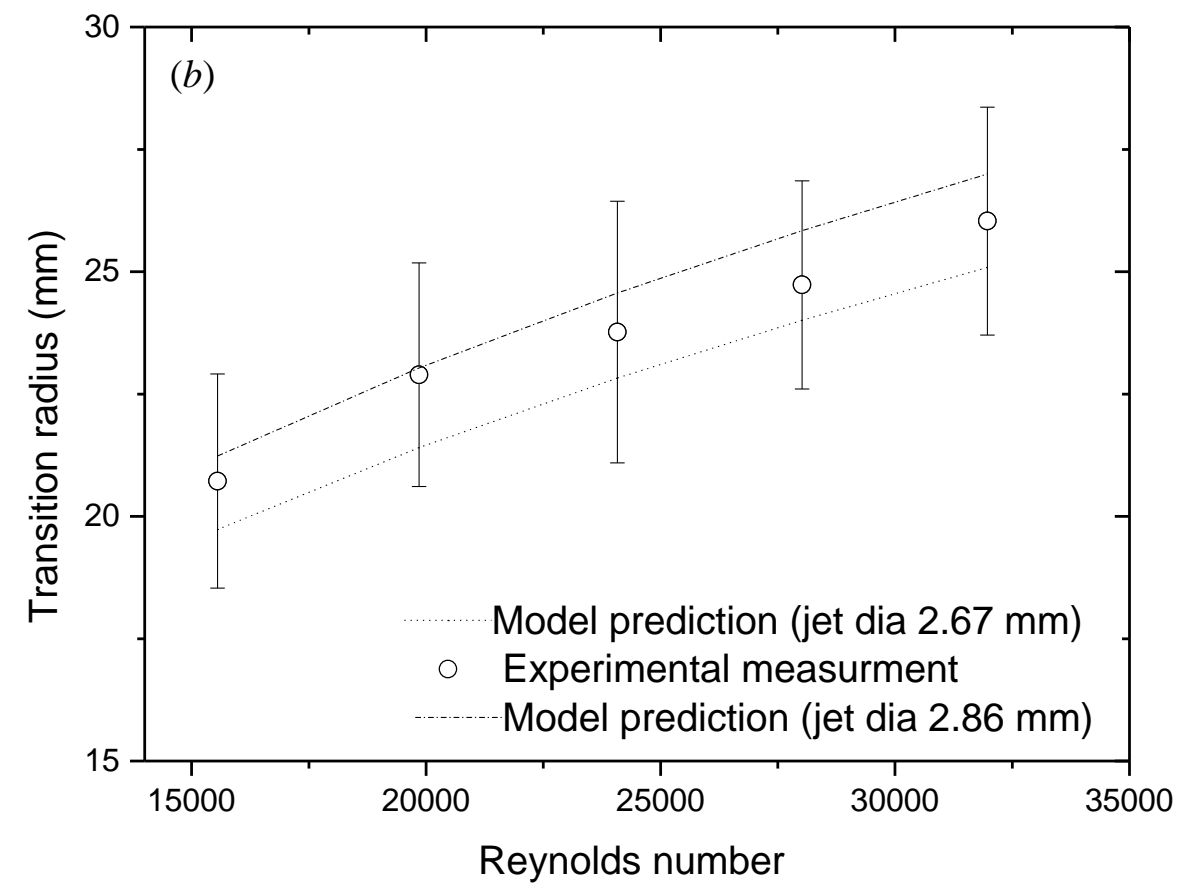

Figure 7 Location of the laminar-turbulent transition, $r_{\mathrm{t}}$ (a) Comparison of this work with existing correlations: $(b)$ Experimental observations of the critical radius for wave breakup alongside the model predictions, Equation (40). The error bars show the standard error of the measured transition radius. 

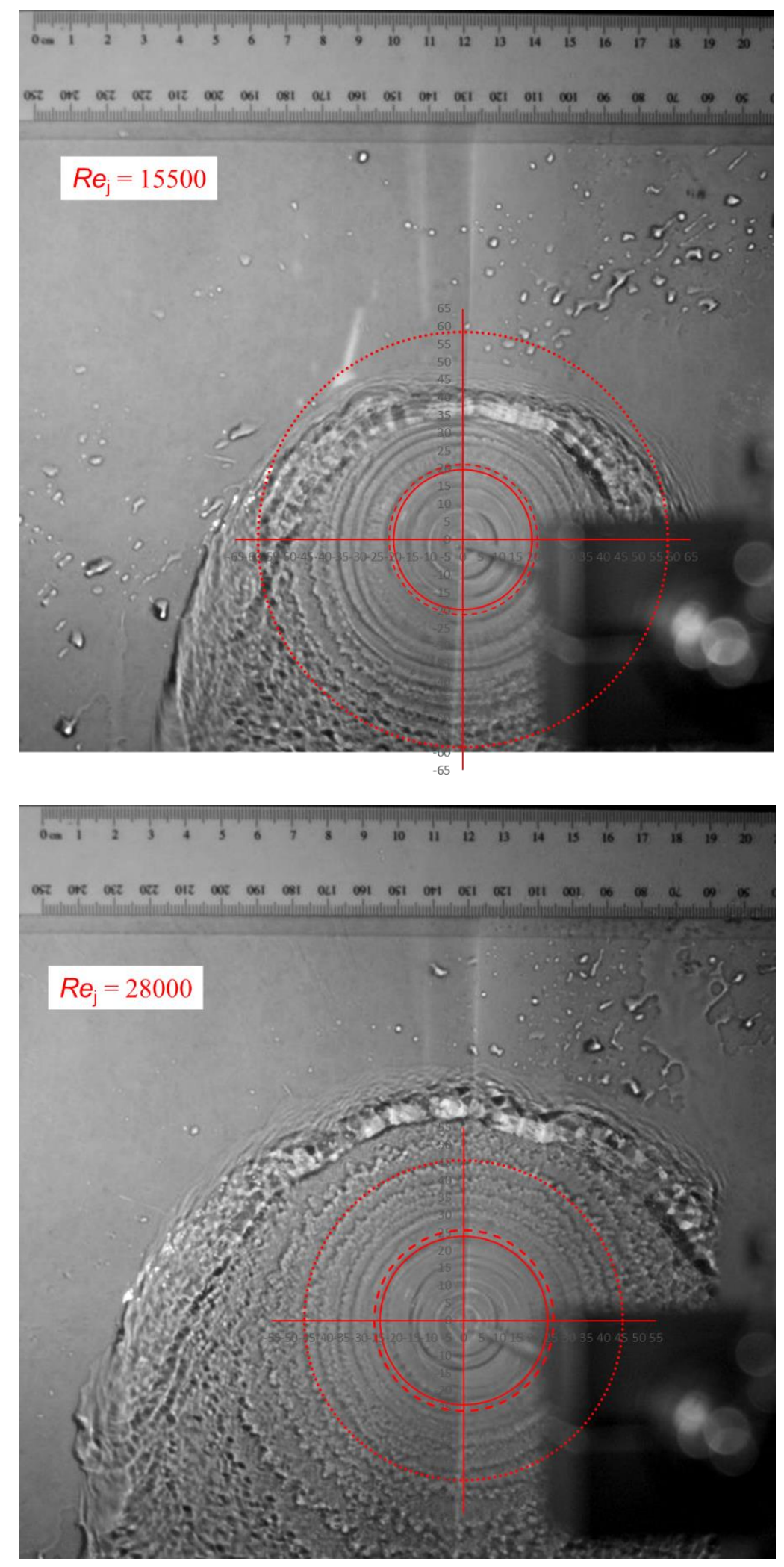

Figure 8 Photographs of the RFZ and draining films obtained with perpendicular impinging jets, $d=2.667 \mathrm{~mm}$ and (a) $R e_{\mathrm{j}}=15500$ and (b) $R e_{\mathrm{j}}=28000$. Dashed line - this work $(d=2.87 \mathrm{~mm})$, solid line - this work $(d=2.67 \mathrm{~mm})$, Equation $(40)$; dotted line - Liu et al. (1991) correlation, Equation (66). 

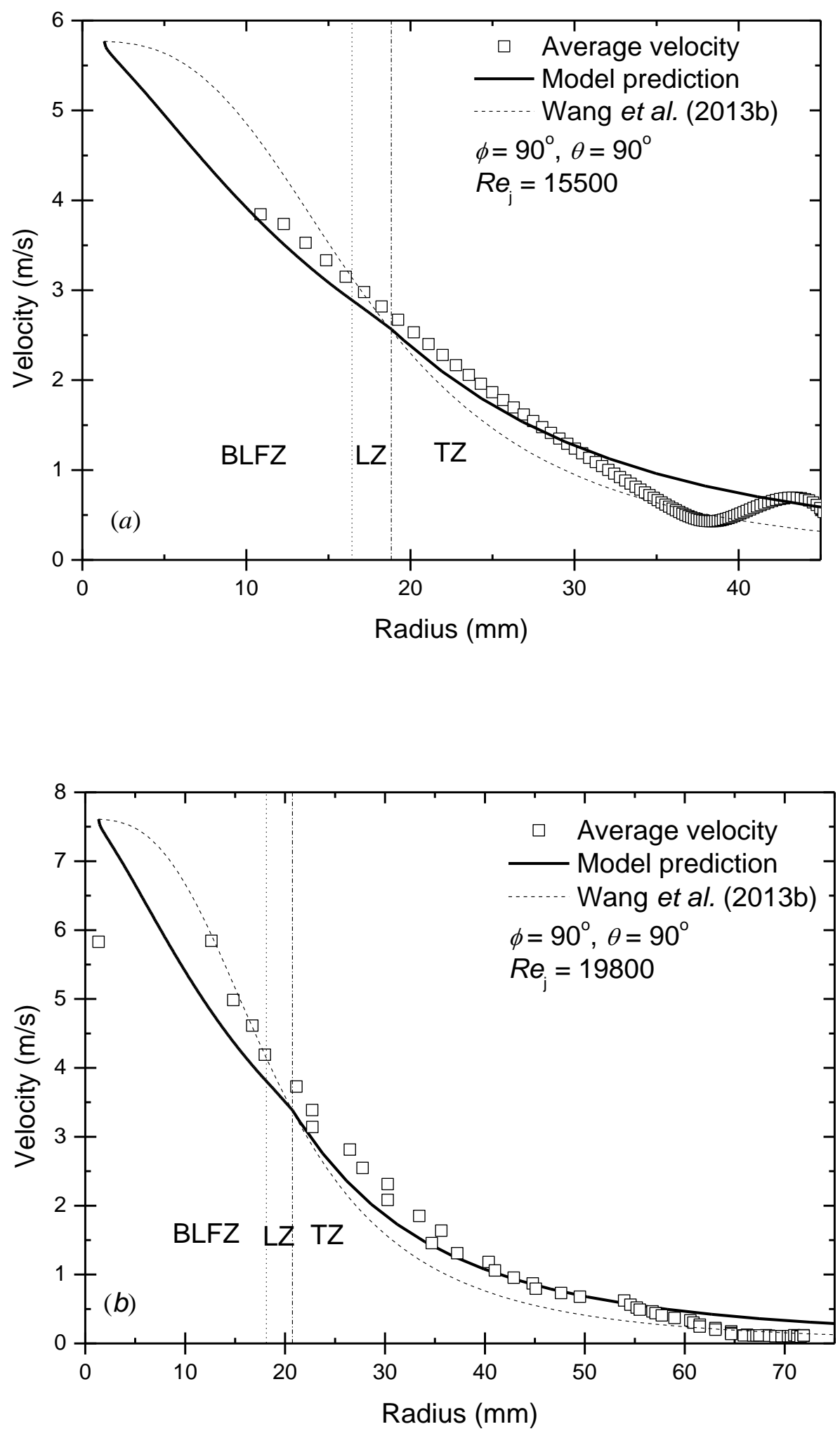

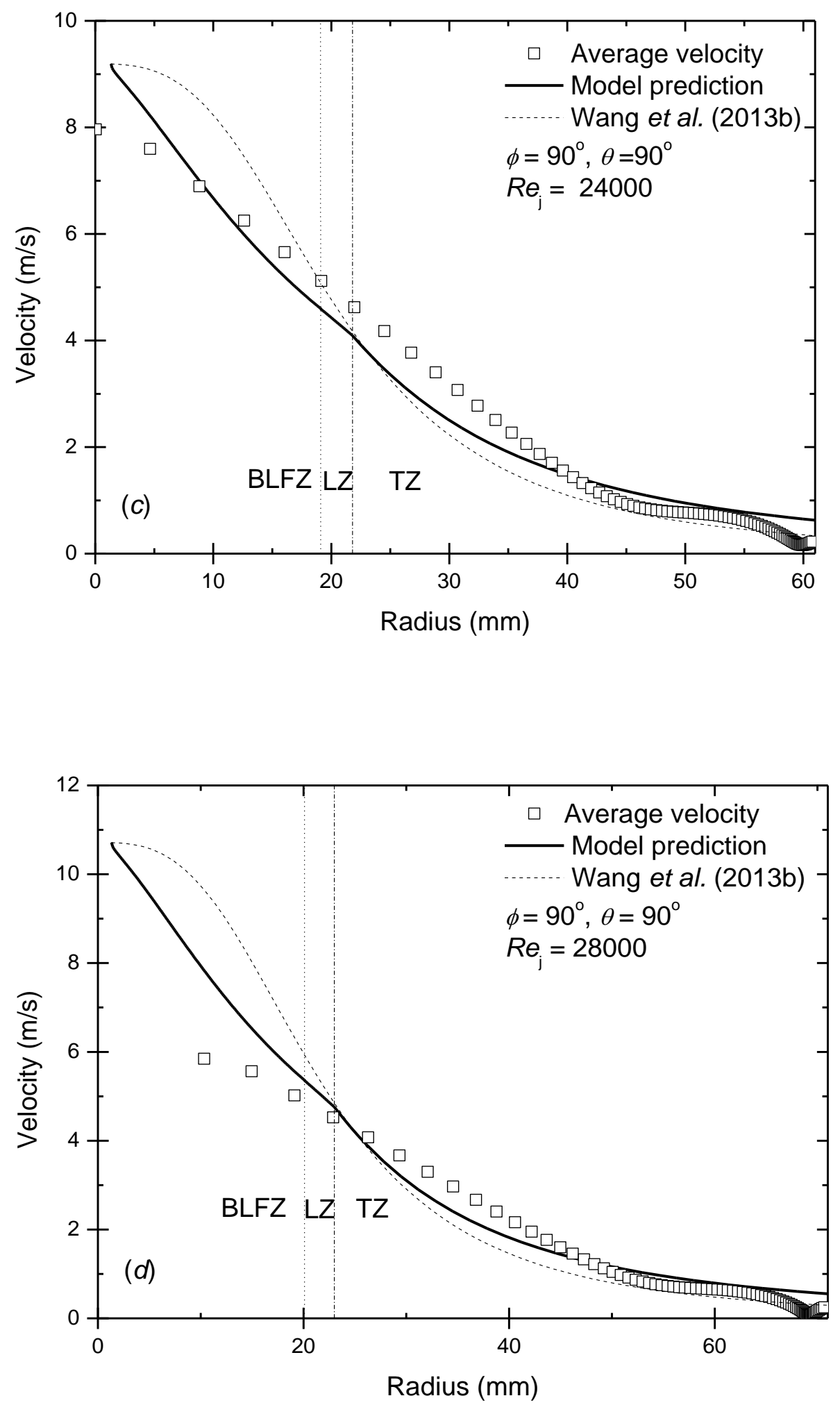


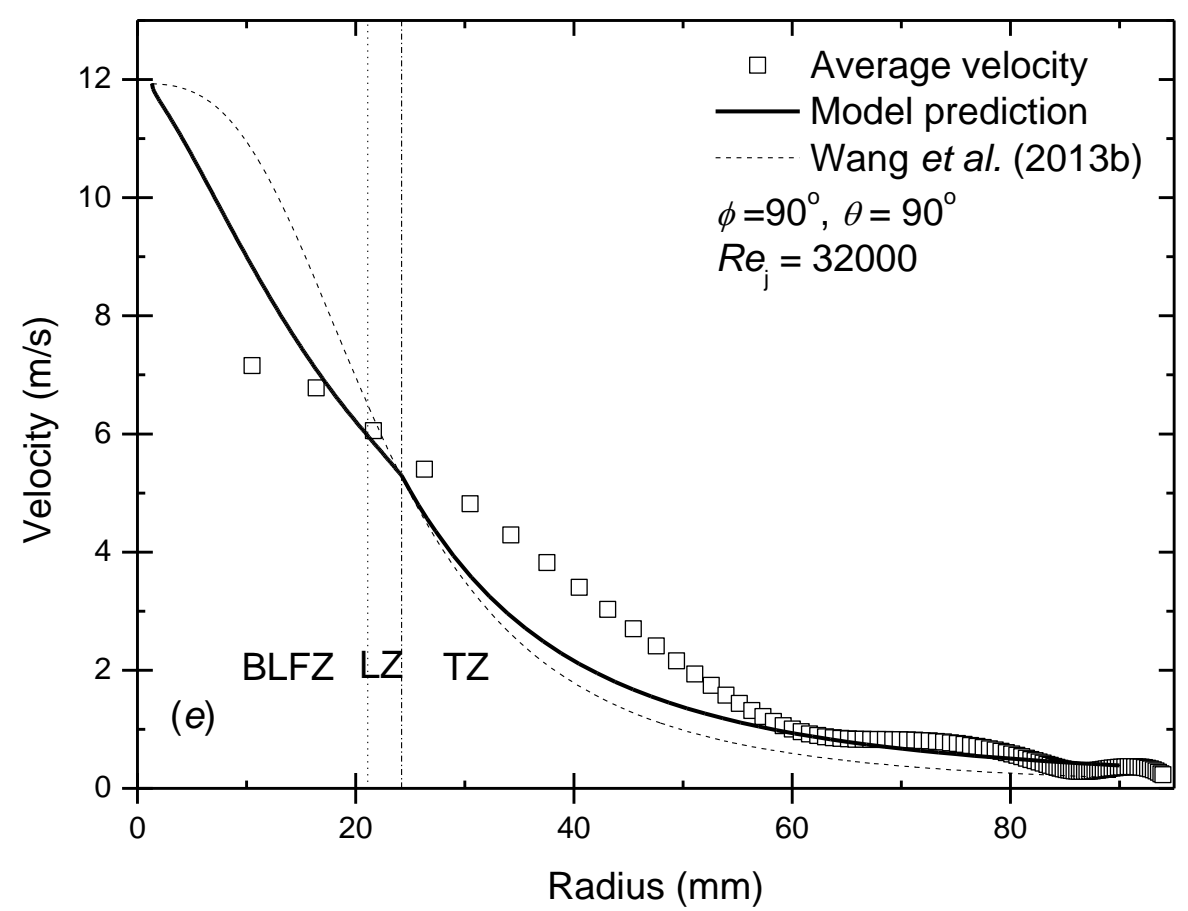

Figure 9 Comparison of experimental estimates of the average velocity $U_{a v}$ obtained from video analysis of the formation of the RFZ with the present model. Vertical glass plate, nozzle diameter $2.667 \mathrm{~mm}, Q=$ (a) $1.95 \mathrm{dm}^{3} / \mathrm{min}$, (b) $2.49 \mathrm{dm}^{3} / \mathrm{min}$, (c) 3.01 $\mathrm{dm}^{3} / \min ,(d) 3.51 \mathrm{dm}^{3} / \mathrm{min}$, and $(e) 4.01 \mathrm{dm}^{3} / \mathrm{min}$. 

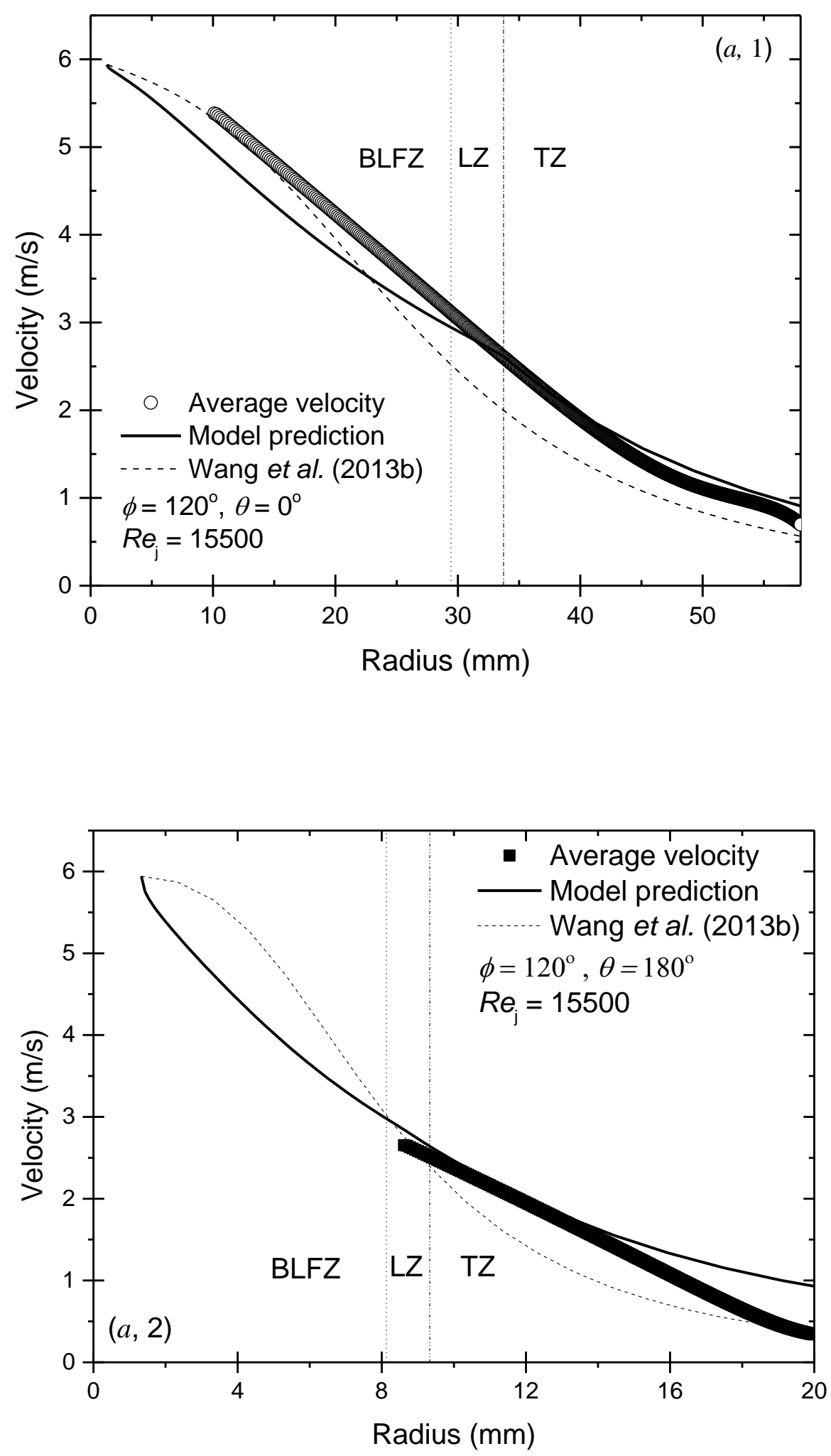

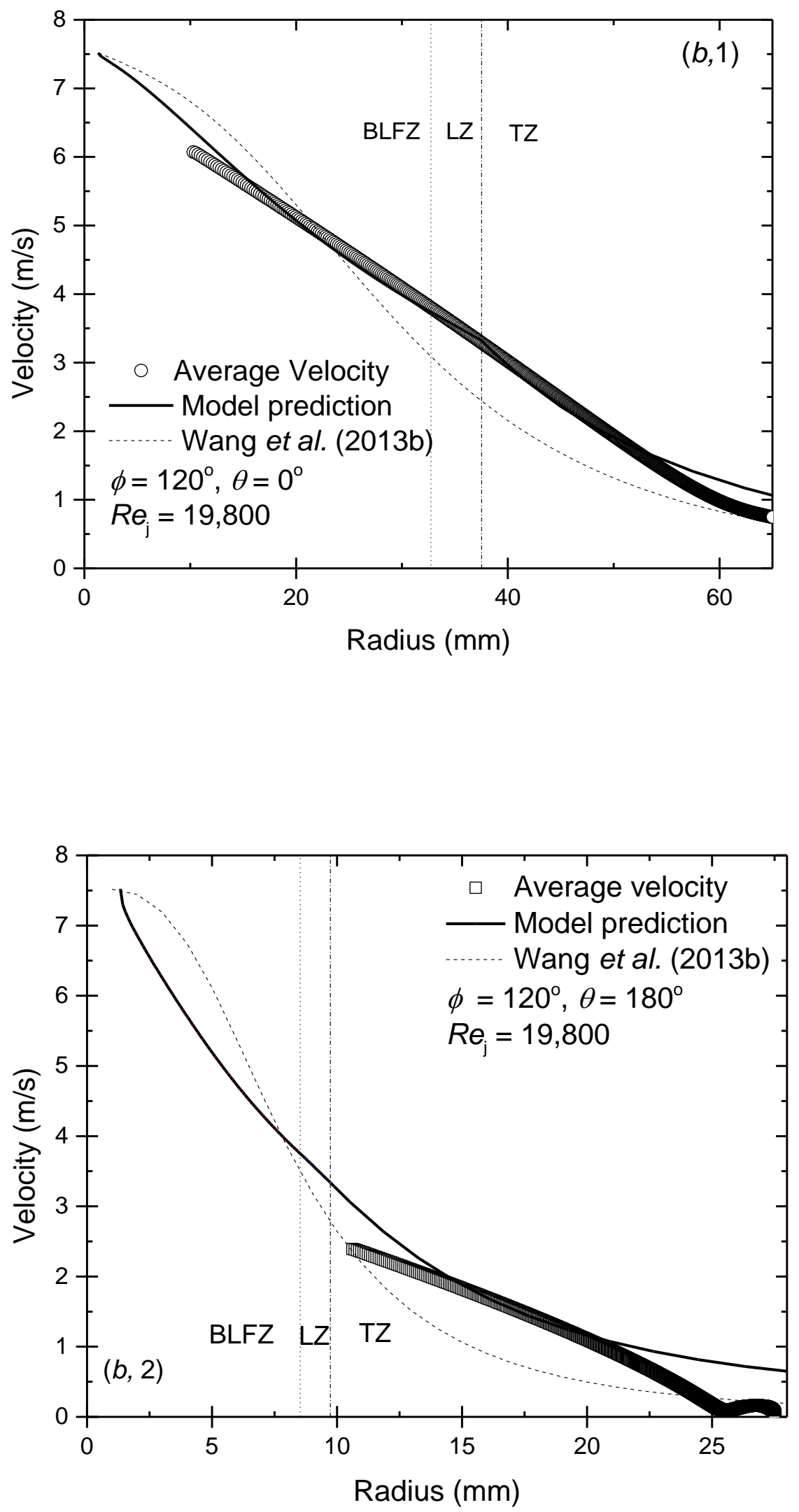

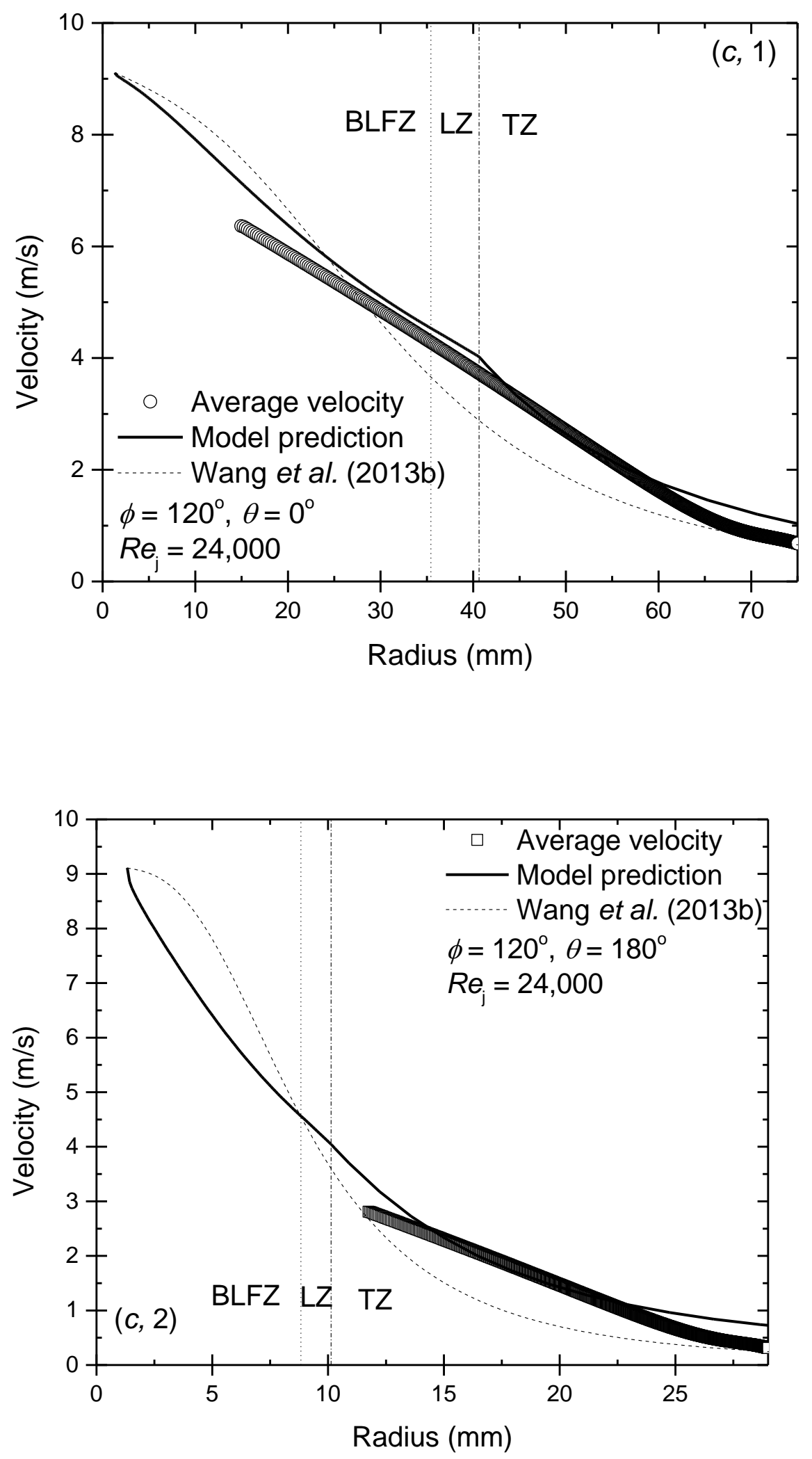

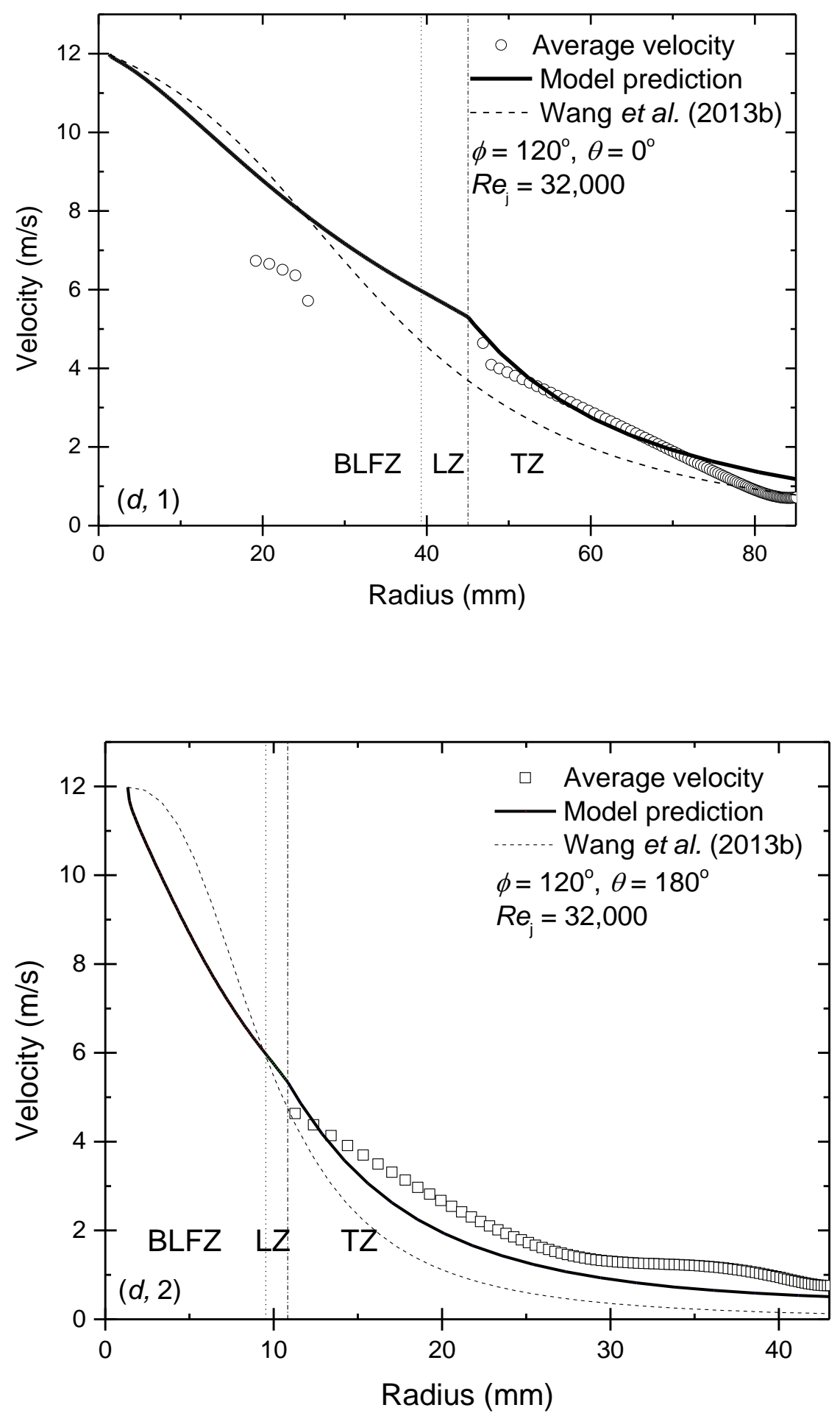

Figure 10 Comparison of estimated average velocity with model predictions for an obliquely impinging water jet $\left(\phi=120^{\circ}\right)$ on a vertical Perspex ${ }^{\mathrm{TM}}$ plate, nozzle diameter 2.667 $\mathrm{mm}$, for $Q=$ (a) $1.99 \mathrm{dm}^{3} / \mathrm{min}$, (b) $2.52 \mathrm{dm}^{3} / \mathrm{min}$; (c) $3.05 \mathrm{dm}^{3} / \mathrm{min}$; (d) $4.01 \mathrm{dm}^{3} / \mathrm{min}$; (1) $\theta=0^{\circ}$, (2) $\theta=180^{\circ}$. 

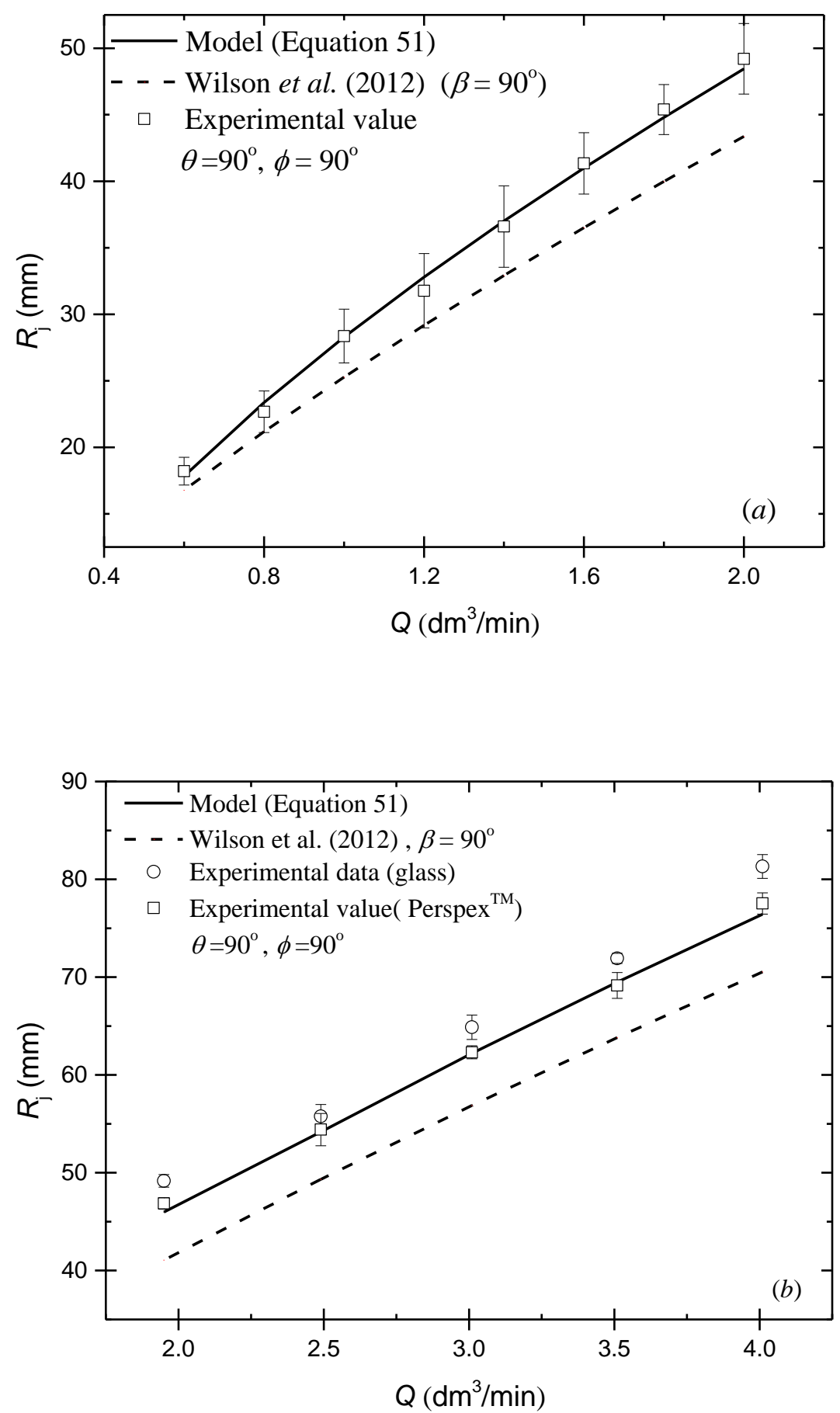

Figure 11 Location of film jump for a jet impinging perpendicularly on a vertical surface at $\theta$ $=90^{\circ}$. Comparison of measured values and the model, Equation (51) for (a) $0.6 \leq Q \leq$ $2 \mathrm{dm}^{3} / \min , d=2 \mathrm{~mm}$, and (b) $1.95 \leq Q \leq 4.01 \mathrm{dm}^{3} / \mathrm{min}, d=2.667 \mathrm{~mm}$. 


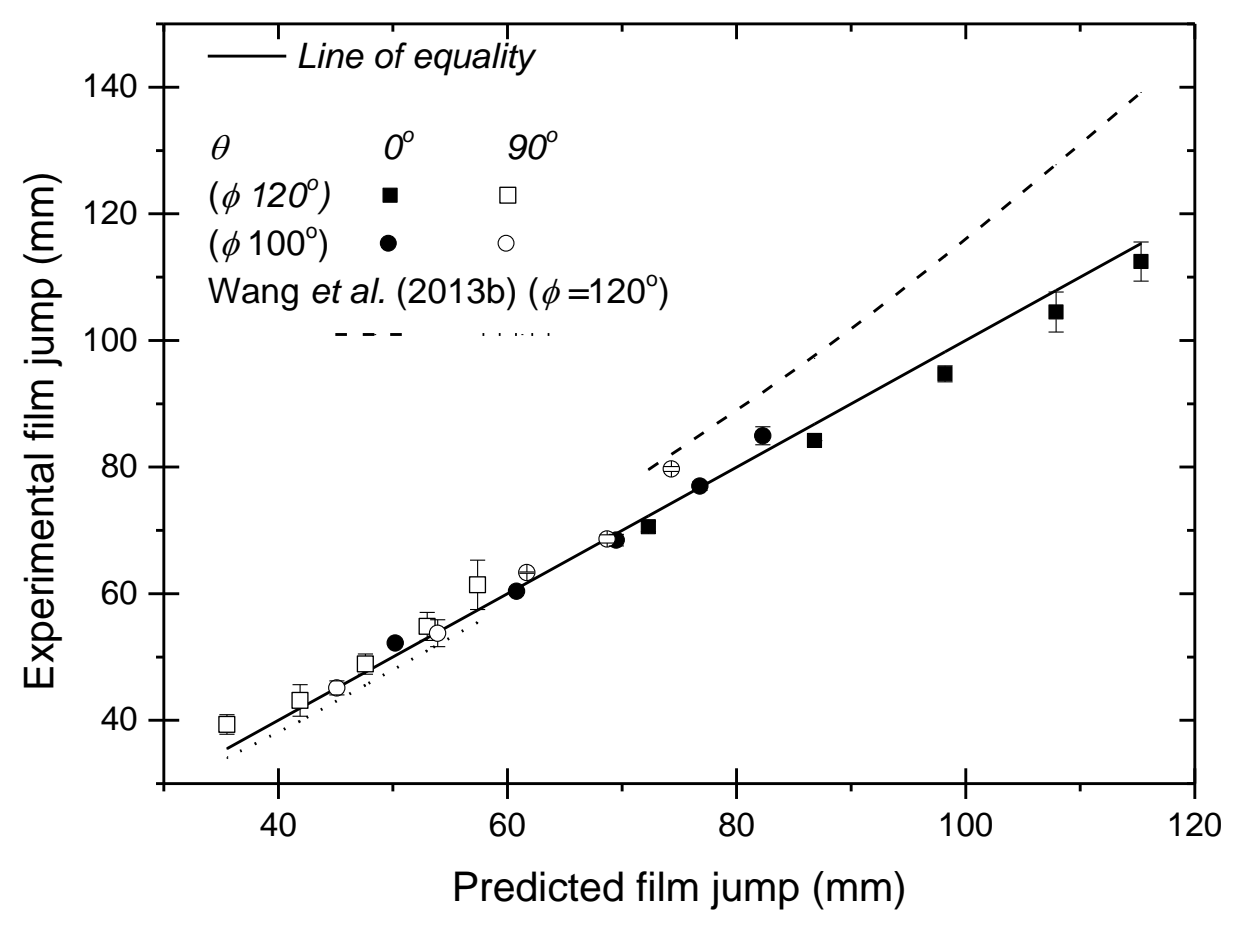

Figure 12 Location of the film jump for a jet impinging obliquely on a vertical surface. Comparison of measured and predicted values of $R_{\mathrm{j}}$ at $\theta=0^{\circ}$ and $90^{\circ}$. Predictions are presented for the present model and that of Wang et al. (2013b). $d=2.667 \mathrm{~mm}, Q=$ $1.95-4.01 \mathrm{dm}^{3} / \mathrm{min}$ : angle of impingement $\phi=100^{\circ}$ and $120^{\circ}$. 


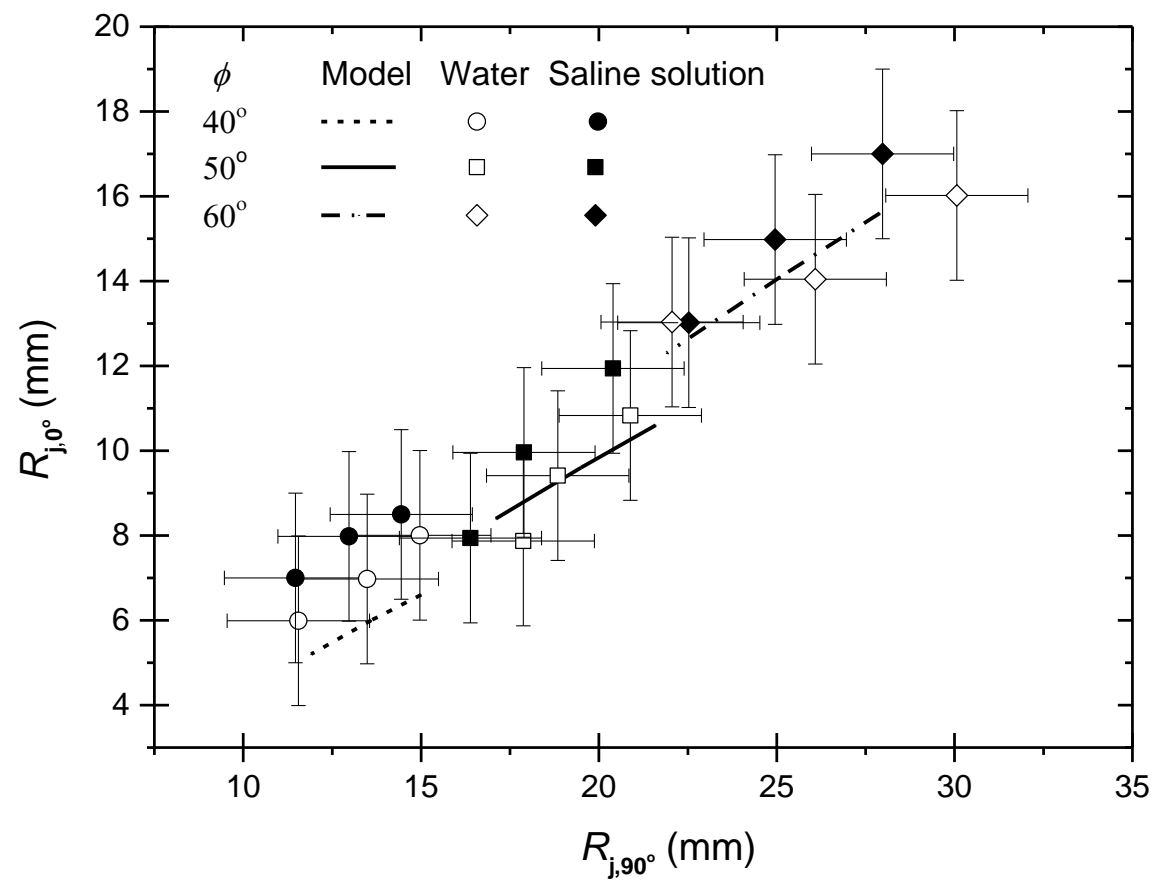

Figure 13 Every man's fluid mechanics: location of film jump at $\theta=90^{\circ}$ and $0^{\circ}$ for $Q=1,1.2$ and $1.4 \mathrm{dm}^{3} / \mathrm{min}$ and notional angles of impingement $40^{\circ}, 50^{\circ}$ and $60^{\circ}$ (Equation 50). Data taken from Hodgson and Smith (2014). Liquid temperature $37^{\circ} \mathrm{C}, d=2 \mathrm{~mm}$ and $R e_{\mathrm{j}}=10600,12700$, and 14 900. Loci show predictions from this work. 


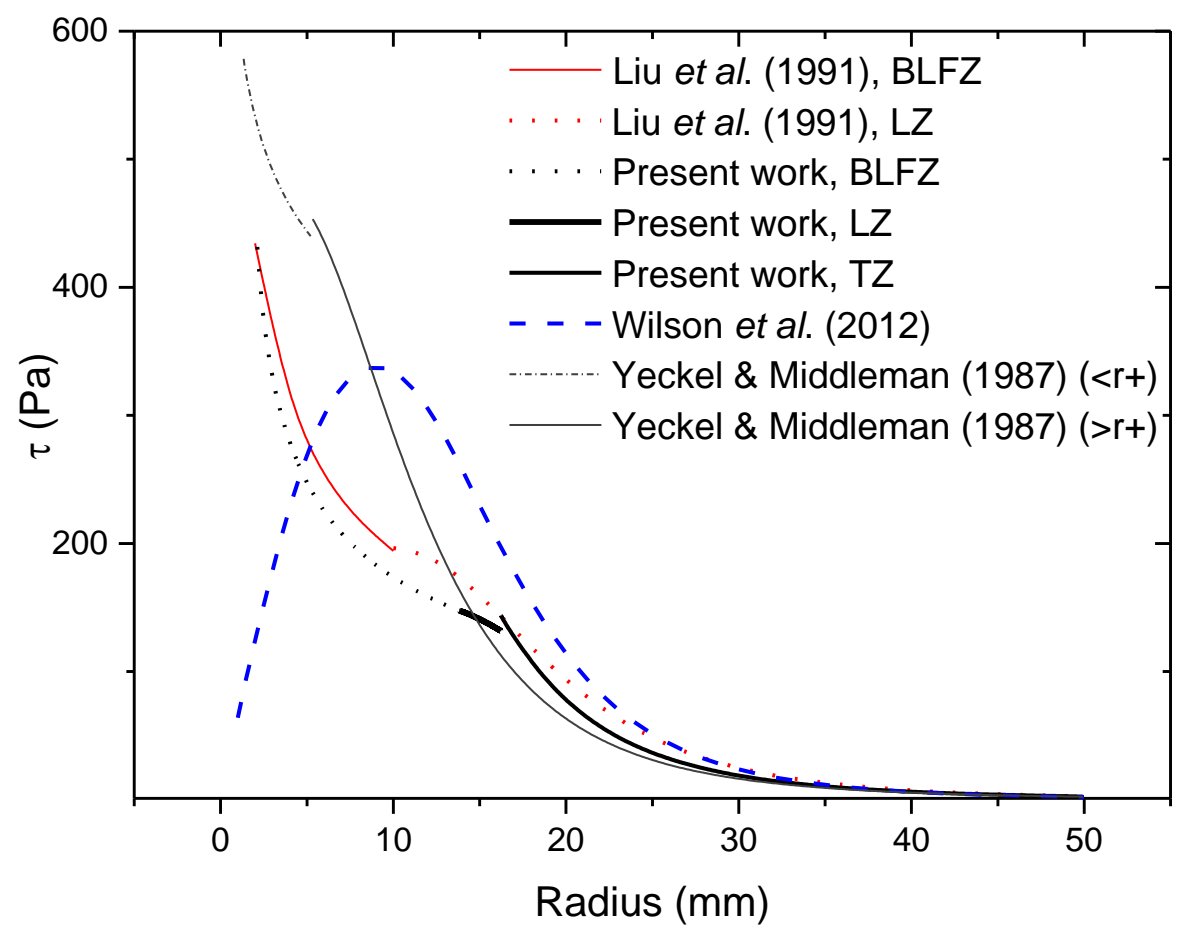

Figure 14 Comparison of the estimated wall shear stress distributions for a jet with $d=2 \mathrm{~mm}$, $R e_{\mathrm{j}}=21000$, flow rate $2 \mathrm{dm}^{3} \mathrm{~min}^{-1}, \theta=90^{\circ}$. 